\title{
Entropy formulation for fractal conservation laws
}

\author{
Nathaël Alibaud
}

$3 / 03 / 2006$

Université Montpellier II, Département de mathématiques, CC 051, Place E. Bataillon, 34095

Montpellier cedex 5, France

E-mail:alibaud@math.univ-montp2.fr

\begin{abstract}
Using an integral formula of Droniou and Imbert (2005) for the fractional Laplacian, we define an entropy formulation for fractal conservation laws with pure fractional diffusion of order $\lambda \in] 0,1]$. This allows to show the existence and the uniqueness of a solution in the $L^{\infty}$ framework. We also establish a result of controled speed of propagation that generalizes the finite propagation speed result of scalar conservation laws. We finally let the non-local term vanish to approximate solutions of scalar conservation laws, with optimal error estimates for $B V$ initial conditions as Kuznecov (1976) for $\lambda=2$ and Droniou (2003) for $\lambda \in] 1,2]$.
\end{abstract}

Keywords: Fractional Laplacian, fractal conservation laws, entropy formulation, vanishing viscosity method, error estimates.

Mathematics Subject Classification: 35B30, 35L65, 35L82, 35S10, 35S30.

\section{Introduction}

We study the fractal conservation law

$$
\begin{cases}\partial_{t} u(t, x)+\operatorname{div}(f(u))(t, x)+g[u(t, .)](x)=0 & t>0, x \in \mathbb{R}^{N}, \\ u(0, x)=u_{0}(x) & x \in \mathbb{R}^{N},\end{cases}
$$

where $f=\left(f_{1}, \ldots, f_{N}\right)$ is locally Lipschitz-continuous from $\mathbb{R}$ to $\mathbb{R}^{N}, u_{0} \in L^{\infty}\left(\mathbb{R}^{N}\right)$ and $g$ is the fractional power of order $\lambda / 2$ of the Laplacian with $\lambda \in] 0,1]$. That is to say, $g$ is the non-local operator defined through the Fourier transform by

$$
\mathcal{F}(g[u(t, .)])(\xi)=|\xi|^{\lambda} \mathcal{F}(u(t, .))(\xi) .
$$

Remark 1.1. We could also very well study equations with source term $h$ and such that $f$ and $h$ depend on $(t, x, u)$. All the methods used in this paper would apply, but this would lead to more technical difficulties and for the sake of clarity, we have chosen to present only the framework above. 
The well-posedness of the pure scalar conservation law, namely the Cauchy problem

$$
\begin{cases}\partial_{t} u(t, x)+\operatorname{div}(f(u))(t, x)=0 & t>0, x \in \mathbb{R}^{N}, \\ u(0, x)=u_{0}(x) & x \in \mathbb{R}^{N},\end{cases}
$$

is well-known since the work of Kruzhkov [11], thanks to the notion of entropy solution. In this paper, we define a notion of entropy solution for (1.1) that allows to solve (1.1) in the $L^{\infty}$ framework. We then consider the problem

$$
\begin{cases}\partial_{t} u^{\varepsilon}(t, x)+\operatorname{div}\left(f\left(u^{\varepsilon}\right)\right)(t, x)+\varepsilon g\left[u^{\varepsilon}(t, .)\right](x)=0 & t>0, x \in \mathbb{R}^{N}, \\ u^{\varepsilon}(0, x)=u_{0}(x) & x \in \mathbb{R}^{N}\end{cases}
$$

and we show that $u^{\varepsilon}$ converges, as $\varepsilon \rightarrow 0$, to the (entropy) solution $u$ of (1.2).

The interest of Equation (1.1) was pointed out to us by two papers of Droniou et al. $[7,6]$ which deal with the case $\lambda \in] 1,2]$ (the results of these papers are recalled below). One of their motivations was a preliminary study of equations involved in the theory of detonation in gases $[3,4]$. In fact, $\lambda$ depends on the unknown in the realistic models and is probably not bounded from below by any $\lambda_{0}>1$. Thus, the case $\lambda=1$ is also of interest. Moreover, the general case $\lambda \in] 0,1]$ has many other applications to hydrodynamics, molecular biology, etc [1, 2].

Equation (1.1) constitutes an extension of the classical parabolic equation

$$
\partial_{t} u+\operatorname{div}(f(u))-\triangle u=0,
$$

which corresponds (up to a multiplicative constant) to the case $\lambda=2$. In this case, it is wellknown that the Cauchy problem is well-posed and that the operator $\partial_{t}-\triangle$ has a regularizing effect; (1.3) then is called the parabolic regularization of (1.2) and the use of such a regularization allows to prove the Kruzhkov result. Depending on the value of $\lambda,(1.1)$ should share properties of (1.4) and/or the non-linear hyperbolic equation (1.2). Most of the studies (well-posedness, asymptotic behaviour, etc) are concerned with the range of exponent $\lambda \in] 1,2]$ (see $[1,2,7,6,8$, 10]). In this case, the operator $\partial_{t}+g[$.$] still has a regularizing effect. The first results on this$ subject are probably due to Biler et al. [1] and these results have recently been strengthened in [7], where the existence and the uniqueness of a smooth solution is proved. Let us also refer the reader to $[9,8]$ for the case of Hamilton-Jacobi equations. For $\lambda \in] 0,1]$, the order of the diffusive part is lower than the order of the hyperbolic part; hence, we do not expect any regularizing effect, since it is natural to think that (1.1) could behaves as (1.2). Let us recall that the possibilities of loss of regularity in finite time and of non-uniqueness of weak solutions of the Cauchy problem (1.2) led to the notion of entropy solution of Kruzhkov. The numerical computations of Clavin et al. [3, 4] lead to think that the solutions of (1.1) may also loose some regularity; but, this point is still an open question whose answer does not seem obvious. Neither there is answer to the question of non-uniqueness of weak solutions in a general framework. To our best knowledge, there is only one existence and uniqueness result for (1.1) with $\lambda \leq 1$. It appears in a paper of Biler et al. [1] which deals also with asymptotic behaviour of solutions. They have established the local-in-time (or global with small initial data) existence and uniqueness of a weak solution of the monodimensional fractal Burgers equation $\left(N=1\right.$ and $\left.f=|.|^{2}\right)$ with $\left.\left.\lambda \in\right] 1 / 2,1\right]$ and $u_{0} \in H^{1}(\mathbb{R})$. The proof does not seem adaptable to other dimensions, parameters $\lambda$ or other initial conditions less regular, since the Sobolev imbeddings and the interpolations used to derive the needed energy estimates would be no longer true. 
Following these comments, a good formulation for (1.1), with $\lambda \in] 0,1]$, is probably an entropy formulation, which we have to define. Let us mention that Carrillo [5] has also used an entropy formulation to study a scalar conservation law perturbed by a local degenerate diffusion operator (of the form $-\triangle(b(u))$ ). In our case, the operator $g$ is non-local. Because of this, the inequality (4.16), already mentioned in [8, Lemma 4.1], seems lead to a too weak formulation, namely (4.17). We discuss this issue in Remark 4.2. To find a good formulation (see Definition 2.1), we have used an integral formula for $g$ (see papers of Imbert [9] and of Droniou and Imbert [8] or Theorem 2.1 below).

This notion of entropy solution has allowed to prove the following results for (1.1): wellposedness in the $L^{\infty}$ framework, maximum principle, controled speed of propagation (see Theorem 3.2 which generalizes the finite propagation speed result of scalar conservation laws), $L^{1}$ contraction, non-increase of the $L^{1}$ norm and the $B V$ semi-norm, etc. The existence is proved by a splitting method, as in $[7,6]$ for $\lambda \in] 1,2]$, and the convergence of this method is proved for general $u_{0} \in L^{\infty}\left(\mathbb{R}^{N}\right)$ (in [7], the convergence of the splitting method has been established only for $\left.u_{0} \in L^{\infty}\left(\mathbb{R}^{N}\right) \cap L^{1}\left(\mathbb{R}^{N}\right) \cap B V\left(\mathbb{R}^{N}\right)\right)$. Note that the classical parabolic regularization could also work. As far as the non-local vanishing viscosity method to (1.2) is concerned, the convergence of $u^{\varepsilon}$ is obtained in the general case and optimal error estimates are stated for $B V$ initial conditions, as in [12] for the parabolic regularization of (1.2) and as in [6] for $\lambda \in] 1,2]$. Let us also refer the reader to $[9,8]$, which derive same error estimates (in an appropriate topology) for Hamilton-Jacobi equations.

The rest of the paper is organized as follow. The entropy formulation is given in Section 2 and the main results are stated in Section 3. These results are finally proved in Sections 4-6 (uniqueness and existence for (1.1) and convergence for (1.3), respectively).

\section{Entropy formulation}

To present our formulation for (1.1), we have to recall the following result on $g$.

Theorem 2.1 (Droniou, Imbert 2005). There exists a constant $c_{N}(\lambda)>0$ that only depends on $N$ and $\lambda$ and such that for all $\varphi \in \mathcal{S}\left(\mathbb{R}^{N}\right)$, all $r>0$ and all $x \in \mathbb{R}^{N}$,

$$
g[\varphi](x)=-c_{N}(\lambda) \int_{|z| \geq r} \frac{\varphi(x+z)-\varphi(x)}{|z|^{N+\lambda}} d z-c_{N}(\lambda) \int_{|z| \leq r} \frac{\varphi(x+z)-\varphi(x)-\nabla \varphi(x) . z}{|z|^{N+\lambda}} .
$$

Moreover, when $\lambda \in] 0,1[$ one can take $r=0$.

Remark 2.1. In the sequel, $g: C_{b}^{\infty}\left(\mathbb{R}^{N}\right) \rightarrow C_{b}^{\infty}\left(\mathbb{R}^{N}\right)$ is defined by this formula.

For a proof of this result, see [8, Theorem 2.1]. Here is our entropy formulation for (1.1).

Definition 2.1. Let $u_{0} \in L^{\infty}\left(\mathbb{R}^{N}\right)$. We define an entropy solution to (1.1) as a function $u \in$ $L^{\infty}(] 0, \infty\left[\times \mathbb{R}^{N}\right)$ and such that for all $r>0$, all non-negative $\varphi \in C_{c}^{\infty}\left(\left[0, \infty\left[\times \mathbb{R}^{N}\right)\right.\right.$, all smooth 
convex function $\eta: \mathbb{R} \rightarrow \mathbb{R}$ and all $\phi=\left(\phi_{1}, \ldots, \phi_{N}\right)$ such that $\phi_{i}^{\prime}=\eta^{\prime} f_{i}^{\prime}(i=1, \ldots, N)\left({ }^{1}\right)$,

$$
\begin{gathered}
\int_{0}^{\infty} \int_{\mathbb{R}^{N}}\left(\eta(u) \partial_{t} \varphi+\phi(u) . \nabla \varphi\right) \\
\quad+c_{N}(\lambda) \int_{0}^{\infty} \int_{\mathbb{R}^{N}} \int_{|z| \geq r} \eta^{\prime}(u(t, x)) \frac{u(t, x+z)-u(t, x)}{|z|^{N+\lambda}} \varphi(t, x) d z d x d t \\
+c_{N}(\lambda) \int_{0}^{\infty} \int_{\mathbb{R}^{N}} \int_{|z| \leq r} \eta(u(t, x)) \frac{\varphi(t, x+z)-\varphi(t, x)-\nabla \varphi(t, x) \cdot z}{|z|^{N+\lambda}} d z d x d t+\int_{\mathbb{R}^{N}} \eta\left(u_{0}\right) \varphi(0, .) \geq 0 .
\end{gathered}
$$

Remark 2.2. i) Notice that (2.2) for $r>0$ implies (2.2) for all $r^{\prime}>r$, but not necessarily for $0<r^{\prime}<r$

ii) when $\lambda \in] 0,1[$, the gradient in the third integral term above can be taken out and this gives an equivalent formulation.

Here are some properties of entropy solutions.

Proposition 2.1. i) Classical solutions to (1.1) are entropy solutions;

ii) entropy solutions to (1.1) are weak solutions in the sense that

$$
\int_{0}^{\infty} \int_{\mathbb{R}^{N}}\left(u \partial_{t} \varphi+f(u) \cdot \nabla \varphi-u g[\varphi]\right)+\int_{\mathbb{R}^{N}} u_{0} \varphi(0, .)=0
$$

for all $\varphi \in C_{c}^{\infty}\left(\left[0, \infty\left[\times \mathbb{R}^{N}\right)\right.\right.$;

iii) entropy solutions are continuous with values in $L_{l o c}^{1}\left(\mathbb{R}^{N}\right)$ (i.e. $u$ is a.e. equal to a function belonging to $C\left(\left[0, \infty\left[; L_{l o c}^{1}\left(\mathbb{R}^{N}\right)\right)\right)\right.$;

iv) if $u$ is an entropy solution of (1.1) then $u(0,)=.u_{0}$.

The proofs of ii) and iv) are similar to those used for the pure scalar conservation laws (see [11]), thanks to Theorem 2.1 to treat the fractal part and thanks to iii) to deduce iv). Hence, these proofs are left to the reader. The item iii) will be needed to prove uniqueness in Section 4 . For a first reading, this item could be assumed in Definition 2.1, since an approximating sequence that converges in $C\left([0, T] ; L_{l o c}^{1}\left(\mathbb{R}^{N}\right)\right.$ ) (for all $T>0$ ) will be constructed in Section 5 . Actually, the formulation allows to find item iii) back without the use of this sequence. For the reader's interest, the proof is given in Appendix 7.2. Let us conclude this section with giving the proof of i), which explains how we obtained our formulation (see also Remark 4.2 about the treatment of the fractal part).

Proof of $i)$. Let us assume that $u_{0}$ is smooth and that $u \in C_{b}^{\infty}\left(\left[0, \infty\left[\times \mathbb{R}^{N}\right)\right.\right.$ satisfies (1.1). Since $\eta$ is convex, $\eta(b)-\eta(a) \geq \eta^{\prime}(a)(b-a)$. Hence,

$$
\eta(u(t, x+z))-\eta(u(t, x))-\nabla(\eta(u))(t, x) . z \geq \eta^{\prime}(u(t, x))(u(t, x+z)-u(t, x)-\nabla u(t, x) . z)
$$

\footnotetext{
${ }^{1}$ Let us recall that such a couple $(\eta, \phi)$ is called an entropy-flux pair.
} 
and

$$
\begin{aligned}
\eta^{\prime}(u(t, x)) g[u(t, .)](x) \geq-c_{N}(\lambda) \eta^{\prime}(u(t, x)) \int_{|z| \geq r} \frac{u(t, x+z)-u(t, x)}{|z|^{N+\lambda}} d z & \\
& -c_{N}(\lambda) \int_{|z| \leq r} \frac{\eta(u(t, x+z))-\eta(u(t, x))-\nabla(\eta(u))(t, x)) . z}{|z|^{N+\lambda}} d z .
\end{aligned}
$$

Let us multiply $(1.1)$ by $\eta^{\prime}(u(t, x))$ to get the following entropy inequality:

$$
\begin{aligned}
& \partial_{t}(\eta(u))(t, x)+\operatorname{div}(\phi(u))(t, x)-c_{N}(\lambda) \eta^{\prime}(u(t, x)) \int_{|z| \geq r} \frac{u(t, x+z)-u(t, x)}{|z|^{N+\lambda}} d z \\
& \quad-c_{N}(\lambda) \int_{|z| \leq r} \frac{\eta(u(t, x+z))-\eta(u(t, x))-\nabla(\eta(u))(t, x)) . z}{|z|^{N+\lambda}} d z \leq 0, \quad t>0, x \in \mathbb{R}^{N} .
\end{aligned}
$$

Let us multiply by $\varphi(t, x)$ and, thanks to an integration by parts, let us put the derivatives on this function. Then,

$$
\begin{aligned}
& \int_{0}^{\infty} \int_{\mathbb{R}^{N}}\left(\eta(u) \partial_{t} \varphi+\phi(u) \cdot \nabla \varphi\right) \\
& \quad+c_{N}(\lambda) \int_{0}^{\infty} \int_{\mathbb{R}^{N}} \int_{|z| \geq r} \eta^{\prime}(u(t, x)) \frac{u(t, x+z)-u(t, x)}{|z|^{N+\lambda}} \varphi(t, x) d z d x d t \\
& \quad+c_{N}(\lambda) \int_{0}^{\infty} \int_{\mathbb{R}^{N}} \int_{|z| \leq r} \frac{\eta(u(t, x+z))-\eta(u(t, x))-\nabla(\eta(u))(t, x) \cdot z}{|z|^{N+\lambda}} \varphi(t, x) d z d x d t \\
& +\int_{\mathbb{R}^{N}} \eta\left(u_{0}\right) \varphi(0, .) \geq 0 .
\end{aligned}
$$

Let us now put the fractional derivative on $\varphi$. We let $I$ denote the third term of (2.3). By Taylor's Formula,

$$
\begin{aligned}
I & =c_{N}(\lambda) \int_{0}^{1} \int_{0}^{\infty} \int_{\mathbb{R}^{N}} \int_{|z| \leq r} \frac{(1-\tau) D^{2}(\eta(u))(t, x+\tau z) z \cdot z}{|z|^{N+\lambda}} \varphi(t, x) d z d x d t d \tau, \\
& =c_{N}(\lambda) \int_{0}^{1} \int_{0}^{\infty} \int_{|z| \leq r}(1-\tau)|z|^{-N-\lambda}\left(\int_{\mathbb{R}^{N}} D^{2}(\eta(u))(t, x+\tau z) z . z \varphi(t, x) d x\right) d z d t d \tau .
\end{aligned}
$$

Note that $D^{2}(\eta(u)) z . z=\operatorname{div}_{x}(F)$ where $F=\left(z_{1} \nabla(\eta(u)) . z, \ldots, z_{n} \nabla(\eta(u)) . z\right)$ (here, we let $z_{i}$ denote the coordinates of $z$ w.r.t. the canonic basis of $\left.\mathbb{R}^{N}\right)$. So, an integration by parts gives

$$
\begin{aligned}
I & =-c_{N}(\lambda) \int_{0}^{1} \int_{0}^{\infty} \int_{|z| \leq r}(1-\tau)|z|^{-N-\lambda}\left(\int_{\mathbb{R}^{N}} \nabla(\eta(u))(t, x+\tau z) \cdot z \nabla \varphi(t, x) . z d x\right) d z d t d \tau, \\
& =-c_{N}(\lambda) \int_{0}^{1} \int_{0}^{\infty} \int_{\mathbb{R}^{N}} \int_{|z| \leq r}(1-\tau)|z|^{-N-\lambda} \nabla(\eta(u))(t, x+\tau z) . z \nabla \varphi(t, x) . z d z d x d t d \tau .(2.4)
\end{aligned}
$$

Let us change the variables by $(\tau, t, x, z) \rightarrow(\tau, t, x+\tau z,-z)$ to get

$$
I=-c_{N}(\lambda) \int_{0}^{1} \int_{0}^{\infty} \int_{\mathbb{R}^{N}} \int_{|z| \leq r}(1-\tau)|z|^{-N-\lambda} \nabla(\eta(u))(t, x) . z \nabla \varphi(t, x+\tau z) . z d z d x d t d \tau .
$$

Computing $I$ backward from (2.4) to (2.3) (exchanging the role of $\eta(u)$ and $\varphi$ ) leads finally to (2.2). 


\section{Main results}

Here is our existence and uniqueness result for (1.1).

Theorem 3.1. If $u_{0} \in L^{\infty}\left(\mathbb{R}^{N}\right)$, then there exists a unique entropy solution $u$ to (1.1).

The uniqueness derives from a more precise result which generalizes the finite propagation speed for pure scalar conservation laws. To present it, we have to introduce the kernel of $g$ : $K(t,):.=\mathcal{F}^{-1}\left(e^{-t||^{\lambda}}\right)$ (defined for $t>0$ ). Let us recall that any solution of $\partial_{t} u+g[u]=0$ can be written by the convolution product $u(t,)=.K(t,) * u.(0,$.$) . The most important property$ of $K$ is its non-negativity, which gives a maximum principle for the preceding equation. Such a result is well-known since the work of Lévy [14]. Using $\int_{\mathbb{R}^{N}} K(1,)=.\mathcal{F}(K(1,)).(0)=1$ and the homogeneity property $K(t,)=.t^{-\frac{N}{\lambda}} K\left(1, t^{-\frac{1}{\lambda}}\right.$.), for all $t>0$, we then see that $(K(t, .))_{t>0}$ is an approximate unit as $t \rightarrow 0$. Another important property that will be needed in this paper is the semi-group property: $K(t+s,)=.K(t,) * K.(s,$.$) , for all t>0$ and all $s>0$. Then, we have the following result.

Theorem 3.2 (Finite-infinite propagation speed). Let $u_{0}, v_{0} \in L^{\infty}\left(\mathbb{R}^{N}\right)$. Consider $u$ and $v$ entropy solutions to (1.1) with initial conditions $u_{0}$ and $v_{0}$, respectively. Then, for all $x_{0} \in \mathbb{R}^{N}$, all $t>0$ and all $R>0$,

$$
\int_{B\left(x_{0}, R\right)}|u(t, .)-v(t, .)| \leq \int_{B\left(x_{0}, R+L t\right)} K(t, .) *\left|u_{0}-v_{0}\right|,
$$

where $L$ is a Lipschitz constant of $f$ on $[-m, m]$, with $m=\max \left\{\left\|u_{0}\right\|_{L^{\infty}\left(\mathbb{R}^{N}\right)},\left\|v_{0}\right\|_{L^{\infty}\left(\mathbb{R}^{N}\right)}\right\}$, and $B(x, r)$ denotes the open ball in $\mathbb{R}^{N}$ of center $x$ and radius $r$.

Remark 3.1. i) Notice that this result still holds true for $\lambda \in] 1,2]$.

ii) The infinite propagation speed of (1.1) can be measured the following way: if supp $\left(u_{0}\right)$ is compact then $\int_{B(x, 1)}|u(1,)|=.\mathcal{O}\left(\operatorname{dist}\left(x, \operatorname{supp}\left(u_{0}\right)\right)^{-\lambda}\right)$ (this can be computed by using that $K(1, x) \sim|x|^{-N-\lambda}$, up to a multiplicative constant, as $\left.|x| \rightarrow+\infty\right)$.

Here are other properties of entropy solutions to (1.1), that will be seen in the course of our study.

Proposition 3.1. i) For all $t \geq 0,\|u(t, .)\|_{L^{\infty}\left(\mathbb{R}^{N}\right)} \leq\left\|u_{0}\right\|_{L^{\infty}\left(\mathbb{R}^{N}\right)}$;

ii) if $u_{0} \in L^{\infty}\left(\mathbb{R}^{N}\right) \cap L^{1}\left(\mathbb{R}^{N}\right)$, then so does $u(t,$.$) , for all t>0$, and $u$ is continuous with values in $L^{1}\left(\mathbb{R}^{N}\right)$;

iii) ( $L^{1}$ contraction) if $u_{0}$ and $v_{0}$ belong to $L^{\infty}\left(\mathbb{R}^{N}\right)$ and $u_{0}-v_{0} \in L^{1}\left(\mathbb{R}^{N}\right)$, then for all $t>0$, $u(t,)-.v(t,.) \in L^{1}\left(\mathbb{R}^{N}\right)$ and $\|u(t, .)-v(t, .)\|_{L^{1}\left(\mathbb{R}^{N}\right)} \leq\left\|u_{0}-v_{0}\right\|_{L^{1}\left(\mathbb{R}^{N}\right)} ;$

iv) if $u_{0} \in L^{\infty}\left(\mathbb{R}^{N}\right) \cap B V\left(\mathbb{R}^{N}\right)$, then so does $u(t,$.$) , for all t>0$, with $|u(t, .)|_{B V\left(\mathbb{R}^{N}\right)} \leq$ $\left|u_{0}\right|_{B V\left(\mathbb{R}^{N}\right)}$. 
Remark 3.2. On the one hand, all these properties are consequences of (3.1), except i) which derives from a more precise inequality, where the absolute values are replaced by positive (or negative) parts (this can be seen with the help of semi-entropies of Kruzhkov, namely entropies of the form $\eta_{k}^{+}=(.-k)^{+}$and $\left.\eta_{k}^{-}=(.-k)^{-}\right)$. In fact, this last result allows to establish more generally a comparison principle between entropy solutions to (1.1). On the other hand, as noticed in Remark 5.1, items i)-iv) also are consequences of the splitting method used to prove existence.

Let us conclude this section with our convergence result for (1.3).

Theorem 3.3. Let $u_{0} \in L^{\infty}\left(\mathbb{R}^{N}\right)$. As $\varepsilon \rightarrow 0$, the entropy solution to (1.3) converges in $C\left([0, T] ; L_{\text {loc }}^{1}\left(\mathbb{R}^{N}\right)\right)$, for all $T>0$, to the entropy solution of $(1.2)$. If moreover $u_{0} \in L^{1}\left(\mathbb{R}^{N}\right) \cap$ $B V\left(\mathbb{R}^{N}\right)$, we have the following error estimates: for all $T>0$,

$$
\left\|u^{\varepsilon}-u\right\|_{C\left([0, T] ; L^{1}\left(\mathbb{R}^{N}\right)\right)}=\left\{\begin{array}{cc}
\mathcal{O}(\varepsilon) & \text { if } \quad \lambda \in] 0,1[ \\
\mathcal{O}(\varepsilon|\ln (\varepsilon)|) & \text { if } \quad \lambda=1
\end{array}\right.
$$

Remark 3.3. As remarked in [6] for $\lambda \in] 1,2]$ and in [8] for Hamilton-Jacobi equations, if we take $f=0$ then the solution to $(1.3)$ is $K(\varepsilon .,.) *_{x} u_{0}$, where $*_{x}$ denotes the convolution product w.r.t. the space variable. Then choosing $u_{0}=\mathbf{1}_{[-1,1]^{N}}$, the properties of $K$ allow to see that the error estimates above are optimal.

\section{Uniqueness of the solution}

This section is devoted to the proof of Theorem 3.2. We use the doubling variables technique of Kruzhkov [11].

\subsection{Doubling variables technique}

Consider $u$ and $v$ as functions of the $(t, x)$ - and the $(s, y)$-variables, respectively. Let us recall that the technique consists in combining the equations on $u$ and $v$ and, choosing test functions which forces $(s, y)$ to be closed to $(t, x)$, deducing another equation on $|u-v|$. Define $\operatorname{sign}:=\mathbf{1}_{[0,+\infty[}-\mathbf{1}_{-\infty, 0]}$. Let us consider entropy and flux of the form $\eta_{k}(a)=|a-k|$ and $\phi_{k}(a)=$ $\int_{k}^{a} \operatorname{sign}(\tau-k) f^{\prime}(\tau) d \tau$. Although $\eta_{k}$ and $\phi_{k}$ are not smooth, it can be used in Definition 2.1. Indeed, there exists a sequence of smooth entropies $\left(\eta_{k}^{n}\right)_{n}$ which converges to $\eta_{k}$ locally uniformly on $\mathbb{R}$ and such that, the sequence of derivatives converges to $a \rightarrow \operatorname{sign}(a-k)$ pointwise on $\mathbb{R}$ and stays bounded by 1 . The associated fluxes $a \rightarrow \int_{k}^{a}\left(\eta_{k}^{n}\right)^{\prime}(\tau) f^{\prime}(\tau) d \tau$ tend to $\phi_{k}$ pointwise on $\mathbb{R}$, thanks to the dominated convergence theorem, and $\left|\int_{k}^{a}\left(\eta_{k}^{n}\right)^{\prime}(\tau) f^{\prime}(\tau) d \tau\right| \leq \int_{k}^{a}\left|f^{\prime}\right|(\tau) d \tau$, for all $a \in$ $\mathbb{R}$. The limit $n \rightarrow+\infty$ in (2.2), thanks again to the dominated convergence theorem, then implies that the entropy-flux pair $\left(\eta_{k}, \phi_{k}\right)$ can be used in Definition 2.1. Let $\psi \in C_{c}^{\infty}\left(\left[0, \infty\left[\times\left[0, \infty\left[\times \mathbb{R}^{N} \times\right.\right.\right.\right.\right.$ $\mathbb{R}^{N}$ ) be non-negative. Let us fix $(s, y)$. Let us take $\varphi(t, x)=\psi(t, s, x, y)$ and $\eta=\eta_{k}$ with 
$k=v(s, y)$ in (2.1) and let us integrate w.r.t. $s$ and $y$. We get

$$
\begin{aligned}
& \int_{0}^{\infty} \int_{0}^{\infty} \int_{\mathbb{R}^{N}} \int_{\mathbb{R}^{N}}|u(t, x)-v(s, y)| \partial_{t} \psi(t, s, x, y)+F(u(t, x), v(s, y)) \cdot \nabla_{x} \psi(t, s, x, y) d y d x d s d t \\
+ & c_{N}(\lambda) \int_{0}^{\infty} \int_{0}^{\infty} \int_{\mathbb{R}^{N}} \int_{\mathbb{R}^{N}} \int_{|z| \geq r} \operatorname{sign}(u(t, x)-v(s, y)) \frac{u(t, x+z)-u(t, x)}{|z|^{N+\lambda}} \psi(t, s, x, y) d z d y d x d s d t \\
+ & c_{N}(\lambda) \int_{0}^{\infty} \int_{0}^{\infty} \int_{\mathbb{R}^{N}} \int_{\mathbb{R}^{N}} \int_{|z| \leq r}|u(t, x)-v(s, y)| \\
& \frac{\psi(t, s, x+z, y)-\psi(t, s, x, y)-\nabla_{x} \psi(t, s, x, y) . z}{|z|^{N+\lambda}} d z d y d x d s d t \\
+ & \int_{0}^{\infty} \int_{\mathbb{R}^{N}} \int_{\mathbb{R}^{N}}\left|u_{0}(x)-v(s, y)\right| \psi(0, s, x, y) d y d x d s \geq 0,
\end{aligned}
$$

where $F(a, b):=\phi_{b}(a)=f(\max (a, b))-f(\min (a, b))$ is symmetric. Same operations for $v$, with $k=u(t, x)$, imply that

$$
\begin{aligned}
& \int_{0}^{\infty} \int_{0}^{\infty} \int_{\mathbb{R}^{N}} \int_{\mathbb{R}^{N}}|u(t, x)-v(s, y)| \partial_{s} \psi(t, s, x, y)+F(u(t, x), v(s, y)) . \nabla_{y} \psi(t, s, x, y) d y d x d s d t \\
+ & c_{N}(\lambda) \int_{0}^{\infty} \int_{0}^{\infty} \int_{\mathbb{R}^{N}} \int_{\mathbb{R}^{N}} \int_{|z| \geq r} \operatorname{sign}(v(s, y)-u(t, x)) \frac{v(s, y+z)-v(s, y)}{|z|^{N+\lambda}} \psi(t, s, x, y) d z d y d x d s d t \\
+ & c_{N}(\lambda) \int_{0}^{\infty} \int_{0}^{\infty} \int_{\mathbb{R}^{N}} \int_{\mathbb{R}^{N}} \int_{|z| \leq r}|u(t, x)-v(s, y)| \\
& \frac{\psi(t, s, x, y+z)-\psi(t, s, x, y)-\nabla_{y} \psi(t, s, x, y) . z}{|z|^{N+\lambda}} d z d y d x d s d t \\
+ & \int_{0}^{\infty} \int_{\mathbb{R}^{N}} \int_{\mathbb{R}^{N}}\left|u(t, x)-v_{0}(y)\right| \psi(t, 0, x, y) d y d x d t \geq 0 .
\end{aligned}
$$

Since the function sign is odd, the sum of the second terms of each inequalities above is equal to

$$
\begin{aligned}
c_{N}(\lambda) \int_{0}^{\infty} & \int_{0}^{\infty} \int_{\mathbb{R}^{N}} \int_{\mathbb{R}^{N}} \int_{|z| \geq r} \psi(t, s, x, y) \\
& \operatorname{sign}(u(t, x)-v(s, y)) \frac{(u(t, x+z)-v(s, y+z))-(u(t, x)-v(s, y))}{|z|^{N+\lambda}} d z d y d x d s d t .
\end{aligned}
$$

Since $\operatorname{sign}(b)(a-b) \leq|a|-|b|$, this expression is bounded from above by

$$
\begin{gathered}
c_{N}(\lambda) \int_{0}^{\infty} \int_{0}^{\infty} \int_{\mathbb{R}^{N}} \int_{\mathbb{R}^{N}} \int_{|z| \geq r} \psi(t, s, x, y) \\
\frac{|u(t, x+z)-v(s, y+z)|-|u(t, x)-v(s, y)|}{|z|^{N+\lambda}} d z d y d x d s d t .
\end{gathered}
$$


The sum of (4.1) and (4.2) then implies that

$$
\begin{gathered}
\int_{0}^{\infty} \int_{0}^{\infty} \int_{\mathbb{R}^{N}} \int_{\mathbb{R}^{N}}|u(t, x)-v(s, y)|\left(\partial_{t}+\partial_{s}\right) \psi(t, s, x, y) \\
+F(u(t, x), v(s, y)) \cdot\left(\nabla_{x}+\nabla_{y}\right) \psi(t, s, x, y) d y d x d s d t \\
+c_{N}(\lambda) \int_{0}^{\infty} \int_{0}^{\infty} \int_{\mathbb{R}^{N}} \int_{\mathbb{R}^{N}} \int_{|z| \geq r} \begin{array}{c} 
\\
\end{array}(t, s, x, y) \\
\frac{|u(t, x+z)-v(s, y+z)|-|u(t, x)-v(s, y)|}{|z|^{N+\lambda}} d z d y d x d s d t \\
+c_{N}(\lambda) \int_{0}^{\infty} \int_{0}^{\infty} \int_{\mathbb{R}^{N}} \int_{\mathbb{R}^{N}} \int_{|z| \leq r}|u(t, x)-v(s, y)| \\
\begin{array}{r}
\psi(t, s, x+z, y)+\psi(t, s, x, y+z)-2 \psi(t, s, x, y)-\left(\nabla_{x}+\nabla_{y}\right) \psi(t, s, x, y) . z \\
|z|^{N+\lambda} d z d y d x d s d t
\end{array} \\
\int_{\mathbb{R}^{N}} \int_{\mathbb{R}^{N}}\left|u_{0}(x)-v(s, y)\right| \psi(0, s, x, y) d y d x d s \\
+\int_{0}^{\infty} \int_{\mathbb{R}^{N}} \int_{\mathbb{R}^{N}}\left|u(t, x)-v_{0}(y)\right| \psi(t, 0, x, y) d y d x d t \geq 0 .
\end{gathered}
$$

An integrability argument immediately implies that the third term of (4.3) tends to 0 as $r \rightarrow 0$. We let $I_{r}$ denote the second term of (4.3). To pass to the limit in $I_{r}$, we have to first put the fractional derivative on $\psi$.

$$
\begin{aligned}
I_{r}=c_{N}(\lambda) \int_{0}^{\infty} \int_{0}^{\infty} & \int_{\mathbb{R}^{N}} \int_{\mathbb{R}^{N}} \int_{|z| \geq r}|u(t, x+z)-v(s, y+z)| \frac{\psi(t, s, x, y)}{|z|^{N+\lambda}} d z d y d x d s d t \\
& \quad-c_{N}(\lambda) \int_{0}^{\infty} \int_{0}^{\infty} \int_{\mathbb{R}^{N}} \int_{\mathbb{R}^{N}} \int_{|z| \geq r}|u(t, x)-v(s, y)| \frac{\psi(t, s, x, y)}{|z|^{N+\lambda}} d z d y d x d s d t
\end{aligned}
$$

and changing the variables by $(x, y, z) \rightarrow(x+z, y+z,-z)$ in the first integral,

$$
\begin{gathered}
I_{r}=c_{N}(\lambda) \int_{0}^{\infty} \int_{0}^{\infty} \int_{\mathbb{R}^{N}} \int_{\mathbb{R}^{N}} \int_{|z| \geq r}|u(t, x)-v(s, y)| \frac{\psi(t, s, x+z, y+z)}{|z|^{N+\lambda}} d z d y d x d s d t \\
-c_{N}(\lambda) \int_{0}^{\infty} \int_{0}^{\infty} \int_{\mathbb{R}^{N}} \int_{\mathbb{R}^{N}} \int_{|z| \geq r}|u(t, x)-v(s, y)| \frac{\psi(t, s, x, y)}{|z|^{N+\lambda}} d z d y d x d s d t \\
=c_{N}(\lambda) \int_{0}^{\infty} \int_{0}^{\infty} \int_{\mathbb{R}^{N}} \int_{\mathbb{R}^{N}} \int_{|z| \geq r}|u(t, x)-v(s, y)| \\
\frac{\psi(t, s, x+z, y+z)-\psi(t, s, x, y)}{|z|^{N+\lambda}} d z d y d x d s d t .
\end{gathered}
$$

For $r \leq 1$, let us cut the integral above, according as $|z| \leq 1$ or not, and let us subtract from it

$$
c_{N}(\lambda) \int_{0}^{\infty} \int_{0}^{\infty} \int_{\mathbb{R}^{N}} \int_{\mathbb{R}^{N}} \int_{r \leq|z| \leq 1}|u(t, x)-v(s, y)| \frac{\left(\nabla_{x}+\nabla_{y}\right) \psi(t, s, x, y) . z}{|z|^{N+\lambda}} d z d y d x d s d t .
$$


Note that this integral equals 0 , since its integrand is odd w.r.t. $z$. So

$$
\begin{gathered}
I_{r}=c_{N}(\lambda) \int_{0}^{\infty} \int_{0}^{\infty} \int_{\mathbb{R}^{N}} \int_{\mathbb{R}^{N}} \int_{|z| \geq 1}|u(t, x)-v(s, y)| \\
\frac{\psi(t, s, x+z, y+z)-\psi(t, s, x, y)}{|z|^{N+\lambda}} d z d y d x d s d t \\
+c_{N}(\lambda) \int_{0}^{\infty} \int_{0}^{\infty} \int_{\mathbb{R}^{N}} \int_{\mathbb{R}^{N}} \int_{r \leq|z| \leq 1}|u(t, x)-v(s, y)| \\
\frac{\psi(t, s, x+z, y+z)-\psi(t, s, x, y)-\left(\nabla_{x}+\nabla_{y}\right) \psi(t, s, x, y) . z}{|z|^{N+\lambda}} d z d y d x d s d t .
\end{gathered}
$$

Again by an integrability argument,

$$
\begin{gathered}
\lim _{r \rightarrow 0} I_{r}=c_{N}(\lambda) \int_{0}^{\infty} \int_{0}^{\infty} \int_{\mathbb{R}^{N}} \int_{\mathbb{R}^{N}} \int_{|z| \geq 1}|u(t, x)-v(s, y)| \\
\frac{\psi}{+c_{N}(\lambda) \int_{0}^{\infty} \int_{0}^{\infty} \int_{\mathbb{R}^{N}} \int_{\mathbb{R}^{N}} \int_{|z| \leq 1}|u(t, x)-v(s, y)|} \\
\frac{\psi(t, s, x+z, y+z)-\psi(t, s, x, y)-\left(\nabla_{x}+\nabla_{y}\right) \psi(t, s, x, y) \cdot z}{|z|^{N+\lambda}} d z d y d x d s d t
\end{gathered}
$$

and thanks to Fubini's Theorem,

$$
\begin{aligned}
& \lim _{r \rightarrow 0} I_{r}=\int_{0}^{\infty} \int_{0}^{\infty} \int_{\mathbb{R}^{N}} \int_{\mathbb{R}^{N}}|u(t, x)-v(s, y)|\left(c_{N}(\lambda) \int_{|z| \geq 1} \frac{\psi(t, s, x+z, y+z)-\psi(t, s, x, y)}{|z|^{N+\lambda}} d z\right. \\
& \left.\quad+c_{N}(\lambda) \int_{|z| \leq 1} \frac{\psi(t, s, x+z, y+z)-\psi(t, s, x, y)-\left(\nabla_{x}+\nabla_{y}\right) \psi(t, s, x, y) \cdot z}{|z|^{N+\lambda}} d z\right) d y d x d s d t
\end{aligned}
$$

Passing to the limit as $r \rightarrow 0$ in (4.3),

$$
\begin{array}{r}
\int_{0}^{\infty} \int_{0}^{\infty} \int_{\mathbb{R}^{N}} \int_{\mathbb{R}^{N}}|u(t, x)-v(s, y)|\left(\partial_{t}+\partial_{s}\right) \psi(t, s, x, y) \\
+F(u(t, x), v(s, y)) \cdot\left(\nabla_{x}+\nabla_{y}\right) \psi(t, s, x, y) d y d x d s d t \\
+\int_{0}^{\infty} \int_{0}^{\infty} \int_{\mathbb{R}^{N}} \int_{\mathbb{R}^{N}}|u(t, x)-v(s, y)|\left(c_{N}(\lambda) \int_{|z| \geq 1} \frac{\psi(t, s, x+z, y+z)-\psi(t, s, x, y)}{|z|^{N+\lambda}} d z\right. \\
\left.+c_{N}(\lambda) \int_{|z| \leq 1} \frac{\psi(t, s, x+z, y+z)-\psi(t, s, x, y)-\left(\nabla_{x}+\nabla_{y}\right) \psi(t, s, x, y) \cdot z}{|z|^{N+\lambda}} d z\right) d y d x d s d t \\
+\int_{0}^{\infty} \int_{\mathbb{R}^{N}} \int_{\mathbb{R}^{N}}\left|u_{0}(x)-v(s, y)\right| \psi(0, s, x, y) d y d x d s \\
+\int_{0}^{\infty} \int_{\mathbb{R}^{N}} \int_{\mathbb{R}^{N}}\left|u(t, x)-v_{0}(y)\right| \psi(t, 0, x, y) d y d x d t \geq 0 .
\end{array}
$$


Let us take $\psi(t, s, x, y)=\theta_{\nu}(s-t) \rho_{\mu}(y-x) \phi(t, x)$, where $\theta_{\nu} \in C_{c}^{\infty}(] 0, \nu[)$ and $\rho_{\mu} \in C_{c}^{\infty}\left(B_{\mu}\right)$ (where we let $B_{\mu}$ denote the ball $\left.B(0, \mu)\right)$ are two approximate units and $\phi \in C_{c}^{\infty}\left(\left[0, \infty\left[\times \mathbb{R}^{N}\right)\right.\right.$ is non-negative. Simple computations imply that

$$
\begin{aligned}
\left(\partial_{t}+\partial_{s}\right) \psi(t, s, x, y) & =\theta_{\nu}(s-t) \rho_{\mu}(y-x) \partial_{t} \phi(t, x), \\
\left(\nabla_{x}+\nabla_{y}\right) \psi(t, s, x, y) & =\theta_{\nu}(s-t) \rho_{\mu}(y-x) \nabla \phi(t, x)
\end{aligned}
$$

and, thanks to $(2.1)$,

$$
\begin{aligned}
& c_{N}(\lambda) \int_{|z| \geq 1} \frac{\psi(t, s, x+z, y+z)-\psi(t, s, x, y)}{|z|^{N+\lambda}} d z \\
&+c_{N}(\lambda) \int_{|z| \leq 1} \frac{\psi(t, s, x+z, y+z)-\psi(t, s, x, y)-\left(\nabla_{x}+\nabla_{y}\right) \psi(t, s, x, y) . z}{|z|^{N+\lambda}} d z \\
&=-\theta_{\nu}(s-t) \rho_{\mu}(y-x) g[\phi(t, .)](x) .
\end{aligned}
$$

Moreover, $\theta_{\nu}$ is equal to 0 on $\left.]-\infty, 0\right]$ and $\psi(t, 0, x, y)=0$. By (4.4),

$$
\begin{gathered}
\int_{0}^{\infty} \int_{0}^{\infty} \int_{\mathbb{R}^{N}} \int_{\mathbb{R}^{N}}|u(t, x)-v(s, y)| \theta_{\nu}(s-t) \rho_{\mu}(y-x) \partial_{t} \phi(t, x) \\
+F(u(t, x), v(s, y)) \cdot \theta_{\nu}(s-t) \rho_{\mu}(y-x) \nabla \phi(t, x) \\
-|u(t, x)-v(s, y)| \theta_{\nu}(s-t) \rho_{\mu}(y-x) g[\phi(t, .)](x) d y d x d s d t \\
+\int_{0}^{\infty} \int_{\mathbb{R}^{N}} \int_{\mathbb{R}^{N}}\left|u_{0}(x)-v(s, y)\right| \theta_{\nu}(s) \rho_{\mu}(y-x) \phi(0, x) d y d x d s \geq 0 .
\end{gathered}
$$

We have

$$
F(u(t, x), v(s, y))=f(\max (u(t, x), v(s, y)))-f(\min (u(t, x), v(s, y))) \leq L|u(t, x)-v(s, y)|,
$$

where $L$ is a Lipschitz constant of $f$ on $[-m, m]$ with $m=\max \left\{\|u\|_{L^{\infty}\left(\mathbb{R}^{N}\right)},\|v\|_{L^{\infty}\left(\mathbb{R}^{N}\right)}\right\}$. Then

$$
\begin{array}{r}
\int_{0}^{\infty} \int_{0}^{\infty} \int_{\mathbb{R}^{N}} \int_{\mathbb{R}^{N}}|u(t, x)-v(s, y)| \theta_{\nu}(s-t) \rho_{\mu}(y-x) \\
\left(\partial_{t} \phi(t, x)+L|\nabla \phi(t, x)|-g[\phi(t, .)](x)\right) d y d x d s d t \\
+\int_{0}^{\infty} \int_{\mathbb{R}^{N}} \int_{\mathbb{R}^{N}}\left|u_{0}(x)-v(s, y)\right| \theta_{\nu}(s) \rho_{\mu}(y-x) \phi(0, x) d y d x d s \geq 0 .
\end{array}
$$

We let $I_{\nu, \mu}$ and $J_{\nu, \mu}$ respectively denote the first and the second term of (4.6). Define $\Phi:=$ $\partial_{t} \phi+L|\nabla \phi|-g[\phi]$ and $\omega_{1}(\nu, \mu)=\left|I_{\nu, \mu}-\int_{0}^{\infty} \int_{\mathbb{R}^{N}}\right| u-v|\Phi|$. Let us recall that $\int_{\mathbb{R}^{N}} \rho_{\mu}=1$ and that for all $t \geq 0, \int_{0}^{\infty} \theta_{\nu}(s-t) d s=1$; hence, Fubini's Theorem gives

$$
\int_{0}^{\infty} \int_{\mathbb{R}^{N}}|u-v| \Phi=\int_{0}^{\infty} \int_{0}^{\infty} \int_{\mathbb{R}^{N}} \int_{\mathbb{R}^{N}}|u(t, x)-v(t, x)| \theta_{\nu}(s-t) \rho_{\mu}(y-x) \Phi(t, x) d y d x d s d t .
$$

Since ||$a|-| b|| \leq|a-b|$,

$$
\begin{aligned}
|| u(t, x)-v(s, y) \mid \theta_{\nu}(s-t) \rho_{\mu}(y-x) \Phi(t, x)- & |u(t, x)-v(t, x)| \theta_{\nu}(s-t) \rho_{\mu}(y-x) \Phi(t, x) \mid \\
& \leq|v(t, x)-v(s, y)| \theta_{\nu}(s-t) \rho_{\mu}(y-x)|\Phi(t, x)|
\end{aligned}
$$


for all $(t, s, x, y) \in\left[0, \infty\left[\times\left[0, \infty\left[\times \mathbb{R}^{N} \times \mathbb{R}^{N}\right.\right.\right.\right.$ and

$$
\omega_{1}(\nu, \mu) \leq \int_{0}^{\infty} \int_{0}^{\infty} \int_{\mathbb{R}^{N}} \int_{\mathbb{R}^{N}}|v(t, x)-v(s, y)| \theta_{\nu}(s-t) \rho_{\mu}(y-x)|\Phi(t, x)| d y d x d s d t .
$$

Let us change the variables by $\left(t, x, s^{\prime}, y^{\prime}\right)=(t, x, s-t, y-x)$. Then

$$
\begin{aligned}
\omega_{1}(\nu, \mu) & \leq \int_{0}^{\infty} \int_{0}^{\infty} \int_{\mathbb{R}^{N}} \int_{\mathbb{R}^{N}}\left|v(t, x)-v\left(t+s^{\prime}, x+y^{\prime}\right)\right| \theta_{\nu}\left(s^{\prime}\right) \rho_{\mu}\left(y^{\prime}\right)|\Phi(t, x)| d y^{\prime} d s^{\prime} d x d t \\
& =\int_{0}^{\infty} \int_{\mathbb{R}^{N}}\left\|\left(v-\mathcal{T}_{\left(-s^{\prime},-y^{\prime}\right)} v\right) \Phi\right\|_{L^{1}(] 0, \infty\left[\times \mathbb{R}^{N}\right)} \theta_{\nu}\left(s^{\prime}\right) \rho_{\mu}\left(y^{\prime}\right) d y^{\prime} d s^{\prime},
\end{aligned}
$$

where we have first integrated w.r.t. $(t, x)$ and we let $\mathcal{T}_{h} \varphi$ denote the translated function $\varphi(.-h)$. Let us prove that the function $\left(s^{\prime}, y^{\prime}\right) \in\left[0, \infty\left[\times \mathbb{R}^{N} \rightarrow\left\|\left(v-\mathcal{T}_{\left(-s^{\prime},-y^{\prime}\right)} v\right) \Phi\right\|_{L^{1}(] 0, \infty\left[\times \mathbb{R}^{N}\right)}\right.\right.$ is continuous. Recalling that $v \in L^{\infty}(] 0, \infty\left[\times \mathbb{R}^{N}\right) \subseteq L_{\text {loc }}^{1}(] 0, \infty\left[\times \mathbb{R}^{N}\right)$, the continuity of the translation in $L^{1}$ implies that $\left(s^{\prime}, y^{\prime}\right) \in\left[0, \infty\left[\times \mathbb{R}^{N} \rightarrow \mathcal{T}_{\left(-s^{\prime},-y^{\prime}\right)} v \in L_{l o c}^{1}(] 0, \infty\left[\times \mathbb{R}^{N}\right)\right.\right.$ is continuous. Moreover, Taylor's Formula and Fubini's Theorem applied to (2.1) give

$$
\begin{aligned}
\|\left. g[\phi]\right|_{L^{1}(] 0, \infty\left[\times \mathbb{R}^{N}\right)} \leq c_{N}(\lambda) \int_{0}^{\infty} & \int_{\mathbb{R}^{N}} \int_{z \geq r} \frac{|\phi(t, x+z)|+|\phi(t, x)|}{|z|^{N+\lambda}} d z d x d t \\
& +c_{N}(\lambda) \int_{0}^{1} \int_{0}^{\infty} \int_{\mathbb{R}^{N}} \int_{z \leq r} \frac{(1-\tau)\left|D^{2} \phi(t, x+\tau z)\right|}{|z|^{N-2+\lambda}} d z d x d t d \tau .
\end{aligned}
$$

Integrating first w.r.t. $(t, x)$,

$$
\|g[\phi]\|_{L^{1}(] 0, \infty\left[\times \mathbb{R}^{N}\right)} \leq C\|\phi\|_{L^{1}(] 0, \infty\left[\times \mathbb{R}^{N}\right)}+C\left\|D^{2} \phi\right\|_{L^{1}(] 0, \infty\left[\times \mathbb{R}^{N}\right)^{N^{2}}},
$$

where $C$ is any constant that only depends on $N$ and $\lambda$. We deduce that $g[\phi] \in L^{1}(] 0, \infty\left[\times \mathbb{R}^{N}\right)$ and a fortiori $\Phi \in L^{1}(] 0, \infty\left[\times \mathbb{R}^{N}\right)$. Similar arguments allow to prove that $\Phi \in L^{\infty}(] 0, \infty\left[\times \mathbb{R}^{N}\right)$ and this completes the proof of the continuity of $\left(s^{\prime}, y^{\prime}\right) \rightarrow\left\|\left(v-\mathcal{T}_{\left(-s^{\prime},-y^{\prime}\right)} v\right) \Phi\right\|_{L^{1}(] 0, \infty\left[\times \mathbb{R}^{N}\right)}$. By (4.7) and classically results on approximate units, $\lim _{\nu, \mu \rightarrow 0} \omega_{1}(\nu, \mu)=0$ and we have proved that

$$
\lim _{\nu, \mu \rightarrow 0} I_{\nu, \mu}=\int_{0}^{\infty} \int_{\mathbb{R}^{N}}|u-v|\left(\partial_{t} \phi+L|\nabla \phi|-g[\phi]\right) .
$$

As far as $J_{\nu, \mu}$ is concerned,

$$
\begin{array}{r}
J_{\nu, \mu} \leq \int_{0}^{\infty} \int_{\mathbb{R}^{N}} \int_{\mathbb{R}^{N}}\left|u_{0}(x)-v_{0}(x)\right| \theta_{\nu}(s) \rho_{\mu}(y-x) \phi(0, x) d y d x d s \\
+\int_{0}^{\infty} \int_{\mathbb{R}^{N}} \int_{\mathbb{R}^{N}}\left|v_{0}(x)-v_{0}(y)\right| \theta_{\nu}(s) \rho_{\mu}(y-x) \phi(0, x) d y d x d s \\
\quad+\int_{0}^{\infty} \int_{\mathbb{R}^{N}} \int_{\mathbb{R}^{N}}\left|v_{0}(y)-v(s, y)\right| \theta_{\nu}(s) \rho_{\mu}(y-x) \phi(0, x) d y d x d s \\
\quad \leq \int_{\mathbb{R}^{N}}\left|u_{0}-v_{0}\right| \phi(0, .)+\omega_{2}(\mu)+\omega_{3}(\nu, \mu),
\end{array}
$$

where

$$
\begin{aligned}
& \omega_{2}(\mu)=\int_{\mathbb{R}^{N}}\left\|\left(v_{0}-\mathcal{T}_{-y^{\prime}} v_{0}\right) \phi(0, x)\right\|_{L^{1}\left(\mathbb{R}^{N}\right)} \rho_{\mu}\left(y^{\prime}\right) d y^{\prime} \rightarrow 0, \text { as } \mu \rightarrow 0, \\
& \text { and } \omega_{3}(\nu, \mu)=\int_{0}^{\infty}\left\|\left(v_{0}-v(s, .)\right) \rho_{\mu} * \phi(0, .)\right\|_{L^{1}\left(\mathbb{R}^{N}\right)} \theta_{\nu}(s) d s .
\end{aligned}
$$


By Proposition 2.1 items iii) and iv),

$$
\omega_{3}(\nu, \mu) \rightarrow 0 \quad \text { as }(\nu, \mu) \rightarrow(0,0)
$$

and the limit $(\nu, \mu) \rightarrow(0,0)$ in (4.6) implies that for all non-negative $\phi \in C_{c}^{\infty}\left(\left[0, \infty\left[\times \mathbb{R}^{N}\right)\right.\right.$,

$$
\int_{0}^{\infty} \int_{\mathbb{R}^{N}}|u-v|\left(\partial_{t} \phi+L|\nabla \phi|-g[\phi]\right)+\int_{\mathbb{R}^{N}}\left|u_{0}-v_{0}\right| \phi(0, .) \geq 0 .
$$

\subsection{Conclusion}

Let $T>0, x_{0} \in \mathbb{R}^{N}, M>L T, 0<\delta<\frac{M-L T}{L}$ and $\gamma \in C_{c}^{\infty}\left(\mathbb{R}^{+}\right)$non-negative, non-increasing and equal to 1 on $[0, M]$. Let $\Theta \in C_{c}^{\infty}([0, T+\delta[)$ be non-negative. Define

$$
\phi(t, x)=\Theta(t) K(T+\delta-t, .) * \gamma\left(\left|.-x_{0}\right|+L t\right)(x) \quad \text { if } 0 \leq t<T, \quad \phi(t, x)=0 \text { if not. }
$$

Remark 4.1. The use of the parameter $\delta$ and the test-function $\Theta$ of (4.13) will be necessary when proving the item iii) of Proposition 2.1 in Appendix 7.2. The reader who has admitted this property in a first reading can consider that $\delta=0$ and that $\Theta$ is defined by $\Theta(t)=\int_{t}^{\infty} \theta_{\nu}(T-s) d s$.

Note that $\gamma\left(\left|.-x_{0}\right|+L.\right) \in C_{c}^{\infty}\left([0, T+\delta] \times \mathbb{R}^{N}\right)$ since it equals 1 on a neighbourhood of $[0, T+\delta] \times\left\{x_{0}\right\}$. By Lemma 7.1 and the non-negativity of $K, \phi$ belongs to $C_{b}^{\infty}\left(\left[0, \infty\left[\times \mathbb{R}^{N}\right)\right.\right.$ and is non-negative. On the one hand, let us prove that $\phi$ can be used in (4.11) although its support is not compact. By the properties of $K, \phi \in C\left(\left[0, \infty\left[; L^{1}\left(\mathbb{R}^{N}\right)\right) \cap L^{1}(] 0, \infty\left[; W^{2,1}\left(\mathbb{R}^{N}\right)\right)\right.\right.$ and by Lemma 7.1,

$$
\begin{aligned}
\partial_{t} \phi(t, x)-L \Theta(t) K(T+\delta-t, .) * \gamma^{\prime}\left(\left|.-x_{0}\right|+L t\right)(x)-g[\phi(t, .)](x) \\
\quad=\Theta^{\prime}(t) K(T+\delta-t, .) * \gamma\left(\left|.-x_{0}\right|+L t\right)(x),
\end{aligned}
$$

for all $t \in\left[0, T+\delta\left[\right.\right.$ and all $x \in \mathbb{R}^{N}$. Estimate (4.8), derived before, still holds true for all $\phi \in C_{b}^{\infty}\left(\left[0, \infty\left[\times \mathbb{R}^{N}\right) \cap L^{1}(] 0, \infty\left[; W^{2,1}\left(\mathbb{R}^{N}\right)\right)\right.\right.$. We deduce that $\partial_{t} \phi \in L^{1}(] 0, \infty\left[\times \mathbb{R}^{N}\right)$, thanks to (4.12), and that $g$ is continuous from $C_{b}^{\infty}\left(\left[0, \infty\left[\times \mathbb{R}^{N}\right) \cap L^{1}(] 0, \infty\left[; W^{2,1}\left(\mathbb{R}^{N}\right)\right)\right.\right.$, endowed with the norm of $L^{1}(] 0, \infty\left[; W^{2,1}\left(\mathbb{R}^{N}\right)\right)$, into $L^{1}(] 0, \infty\left[\times \mathbb{R}^{N}\right)$. The density of $C_{c}^{\infty}\left(\left[0, \infty\left[\times \mathbb{R}^{N}\right)\right.\right.$ in

$$
E=\left\{w: w \in C\left(\left[0, \infty\left[; L^{1}\left(\mathbb{R}^{N}\right)\right) \cap L^{1}(] 0, \infty\left[; W^{2,1}\left(\mathbb{R}^{N}\right)\right) \text { and } \partial_{t} w \in L^{1}(] 0, \infty\left[\times \mathbb{R}^{N}\right)\right\}\right.\right.
$$

then implies that $\phi$ can be used in (4.11). On the other hand,

$$
\begin{aligned}
|\nabla \phi(t, x)|=\mid \Theta(t) K(T+\delta-t, .) *\left(\frac{.-x_{0}}{\left|\cdot-x_{0}\right|} \gamma^{\prime}\right. & \left.\left(\left|.-x_{0}\right|+L t\right)\right)(x) \mid \\
& \leq-\Theta(t) K(T+\delta-t, .) * \gamma^{\prime}\left(\left|.-x_{0}\right|+L t\right)(x)
\end{aligned}
$$

because $\gamma$ is non-increasing and by (4.12),

$$
\partial_{t} \phi(t, x)+L|\nabla \phi(t, x)|-g[\phi(t, .)](x) \leq \Theta^{\prime}(t) K(T+\delta-t, .) * \gamma\left(\left|.-x_{0}\right|+L t\right)(x),
$$

for all $t \in\left[0, T+\delta\left[\right.\right.$ and all $x \in \mathbb{R}^{N}$. Let us take

$$
\Theta(t)=\int_{t}^{\infty} \theta_{\nu}(s-T) d s,
$$


where $\theta_{\nu}$ is an approximate unit as before with $0<\nu \leq \delta$ (in order that $\operatorname{supp}(\Theta) \subseteq[0, T+\delta[$ ). We get $\Theta(0)=1$, for $\nu$ sufficiently small, and $\Theta^{\prime}(t)=-\theta_{\nu}(t-T)$. By (4.11),

$$
\begin{aligned}
\int_{0}^{T+\delta} \theta_{\nu}(t-T)\left(\int_{\mathbb{R}^{N}}|u(t, .)-v(t, .)| K(T+\right. & \left.\delta-t, .) * \gamma\left(\left|.-x_{0}\right|+L t\right)\right) d t \\
& \leq \int_{\mathbb{R}^{N}}\left|u_{0}-v_{0}\right| K(T+\delta, .) * \gamma\left(\left|.-x_{0}\right|\right) .
\end{aligned}
$$

By the properties of $K, t \in\left[0, T+\delta\left[\rightarrow K(T+\delta-t,). * \gamma\left(\left|.-x_{0}\right|+L t\right) \in L^{\infty}\left(\mathbb{R}^{N}\right)\right.\right.$ is continuous and $\left.\sup _{t \in[0, T+\delta[}|| K(T+\delta-t,). * \gamma\left(\left|.-x_{0}\right|+L t\right)\right|_{L^{1}\left(\mathbb{R}^{N} \backslash B_{R}\right)} \rightarrow 0$ as $R \rightarrow+\infty$. By Proposition 2.1 item iii), the limit as $\nu \rightarrow 0$ in (4.14) gives

$$
\int_{\mathbb{R}^{N}}|u(T, .)-v(T, .)| K(\delta, .) * \gamma\left(\left|.-x_{0}\right|+L T\right) \leq \int_{\mathbb{R}^{N}}\left|u_{0}-v_{0}\right| K(T+\delta, .) * \gamma\left(\left|.-x_{0}\right|\right) .
$$

Since $K$ is an approximate unit, the limit as $\delta \rightarrow 0$ gives

$$
\begin{aligned}
\int_{\mathbb{R}^{N}}|u(T, .)-v(T, .)| \gamma\left(\left|.-x_{0}\right|+L T\right) & \leq \int_{\mathbb{R}^{N}}\left|u_{0}-v_{0}\right| K(T, .) * \gamma\left(\left|.-x_{0}\right|\right), \\
& =\int_{\mathbb{R}^{N}} \gamma\left(\left|.-x_{0}\right|\right) K(T, .) *\left|u_{0}-v_{0}\right| .
\end{aligned}
$$

The last equality is obtained by using Fubini's Theorem and the parity of $K(T,$.$) . For any real$ $R>0$, we take $M=R+L T$. The characteristic function $\mathbf{1}_{B\left(x_{0}, R+L T\right)}$ can be approximated, in $L^{1}\left(\mathbb{R}^{N}\right)$, by functions of the form $\gamma\left(\left|.-x_{0}\right|\right)$, where $\gamma \in C_{c}^{\infty}\left(\mathbb{R}^{+}\right)$is non-negative, nonincreasing and equal to 1 on $[0, M]$. Passing to the limit in the inequality above, we get (3.1) with $m=\max \left\{\|u\|_{L^{\infty}\left(\mathbb{R}^{N}\right)},\|v\|_{L^{\infty}\left(\mathbb{R}^{N}\right)}\right\}$. In fact, this result suffices to prove the uniqueness of an entropy solution to (1.1), and (3.1) with the best constant will be a consequence of Remark 5.1 .

Remark 4.2. Observe that

$$
\text { if } u \text { is smooth then for all entropy } \eta, \eta^{\prime}(u) g[u] \geq g[\eta(u)] \text {. }
$$

This leads to the following formulation for (1.1) which is more simple than (2.2): for all nonnegative $\varphi \in C_{c}^{\infty}\left(\left[0, \infty\left[\times \mathbb{R}^{N}\right)\right.\right.$ and all entropy-flux pair $(\eta, \phi)$,

$$
\int_{0}^{\infty} \int_{\mathbb{R}^{N}}\left(\eta(u) \partial_{t} \varphi+\phi(u) . \nabla \varphi+\eta(u) g[\varphi]\right)+\int_{\mathbb{R}^{N}} \eta\left(u_{0}\right) \varphi(0, .) \geq 0 .
$$

Let us explain why we do not have used this formulation that will be called intermediate. It is immediate that "classical $\Rightarrow$ entropy $\Rightarrow$ intermediate $\Rightarrow$ weak" (the second implication derives from the limit $r \rightarrow+\infty$ in (2.2)). Conversely, the question of the implication "intermediate $\Rightarrow$ entropy "is open. It is relied with the possible non-uniqueness of intermediate solutions to (1.1). From a technical viewpoint, (4.17) seems to be unappropriate to use the doubling variable technique. Indeed, the term $\left(g_{x}+g_{y}\right)[\psi]$ corresponding to (4.5) behaves as $g\left[\rho_{\mu}\right]$ (this is easy to see if $u$ and $v$ are in $L^{1}$ and $\left.\phi=1\right)$. Then we can not pass to the limit as $\mu \rightarrow 0$. In the case of a local degenerate diffusion of the form $-\triangle(b(u))$, this problem can be resolved by putting a gradient operator on test-functions, thanks to an integration by parts (see [5]). In our case, this is no tractable since $\left(\left(-\triangle_{x}\right)^{\lambda / 4}+\left(-\triangle_{y}\right)^{\lambda / 4}\right) \psi$ behaves as $(-\triangle)^{\lambda / 4} \rho_{\mu}$. Thus, we should put the operator $g$ on $\eta(u)$, but this need some regularity on $\eta(u)$. Even if $\eta(u)$ is regular enough, the non-locality of $g$ and the doubling variable technic give us a diffusive term in (4.11) which is computed from $|u(x)-v(y)|$ and which can non-vanish even if $u=v$. 


\section{Existence of the solution}

We construct here an entropy solution to (1.1), thus concluding Theorem 3.1. We use a splitting method, as in $[7,6]$ for $\lambda \in] 1,2]$. For $\delta>0$, let us construct $u^{\delta}:\left[0, \infty\left[\times \mathbb{R}^{N} \rightarrow \mathbb{R}\right.\right.$ the following way: we let $u^{\delta}(0,)=.u_{0}$ and, for all even $p$ and all odd $q$, we define by induction

(a) $u^{\delta}$ on $\left.] p \delta,(p+1) \delta\right] \times \mathbb{R}^{N}$ as the solution to $\partial_{t} u^{\delta}+2 g\left[u^{\delta}\right]=0$ with initial condition $u^{\delta}(p \delta,$.$) ,$ that is to say $u^{\delta}(t, x)=K(2(t-p \delta),). * u^{\delta}(p \delta,).(x)$;

(b) $u^{\delta}$ on $\left.] q \delta,(q+1) \delta\right] \times \mathbb{R}^{N}$ as the entropy solution to $\partial_{t} u^{\delta}+2 \operatorname{div}\left(f\left(u^{\delta}\right)\right)=0$ with initial condition $u^{\delta}(q \delta,$.$) .$

It is well-known that both equations above do not increase the $L^{\infty}$ norm and that their solutions are continuous with values in $L_{l o c}^{1}\left(\mathbb{R}^{N}\right)$. We thus have defined $u^{\delta} \in C\left(\left[0, \infty\left[; L_{l o c}^{1}\left(\mathbb{R}^{N}\right)\right)\right.\right.$ such that

$$
\left\|u^{\delta}(t, .)\right\|_{L^{\infty}\left(\mathbb{R}^{N}\right)} \leq\left\|u_{0}\right\|_{L^{\infty}\left(\mathbb{R}^{N}\right)},
$$

for all $\delta>0$ and all $t>0$.

\subsection{Compactness result on the sequence $\left(u^{\delta}\right)_{\delta>0}$}

Let us prove that $\left\{u^{\delta}: \delta>0\right\}$ is relatively compact in $C\left([0, T] ; L_{l o c}^{1}\left(\mathbb{R}^{N}\right)\right.$ ) (for all $T>0$ ). The starting point is the following approximate finite-infinite propagation speed property for $u^{\delta}$ : for all $h \in \mathbb{R}^{N}$ and all $R>0$,

$$
\int_{B_{R}}\left|u^{\delta}((p+1) \delta, .)-\mathcal{T}_{h}\left(u^{\delta}((p+1) \delta, .)\right)\right| \leq \int_{B_{R+L p \delta}} K((p+2) \delta, .) *\left|u_{0}-\mathcal{T}_{h} u_{0}\right|,
$$

for all even $p$ and

$$
\int_{B_{R}}\left|u^{\delta}((q+1) \delta, .)-\mathcal{T}_{h}\left(u^{\delta}((q+1) \delta, .)\right)\right| \leq \int_{B_{R+L(q+1) \delta}} K((q+1) \delta, .) *\left|u_{0}-\mathcal{T}_{h} u_{0}\right|,
$$

for all odd $q$, where $L$ is defined as in Theorem 3.2 (with $v_{0}=\mathcal{T}_{h} u_{0}$ ). The proofs of (5.2) and (5.3) are very similar and we give only the proof of (5.2). It is immediate that (5.2) holds true for $p=0$ and in what follows, we assume that $p \geq 2$. For all integer $n$ such that $0 \leq 2 n \leq p$, define

$$
I(n)=\int_{B_{R+L 2 n \delta}} K(2(n+1) \delta, .) *\left|u^{\delta}((p-2 n) \delta, .)-\mathcal{T}_{h}\left(u^{\delta}((p-2 n) \delta, .)\right)\right| .
$$

The finite propagation speed for scalar conservation laws and (5.1) imply that for all $y \in \mathbb{R}^{N}$ and all $0 \leq 2(n+1) \leq p$,

$$
\begin{aligned}
\int_{B_{R+L 2 n \delta}}\left|u^{\delta}((p-2 n) \delta, x-y)-u^{\delta}((p-2 n) \delta, x-y-h)\right| d x \\
\quad \leq \int_{B_{R+L 2 n \delta+L 2 \delta}}\left|u^{\delta}((p-2 n-1) \delta, x-y)-u^{\delta}((p-2 n-1) \delta, x-y-h)\right| d x .
\end{aligned}
$$


Multiplying by $K(2(n+1) \delta, y) \geq 0$ and integrating w.r.t. $y$, Fubini's Theorem gives

$$
\begin{aligned}
\int_{B_{R+L 2 n \delta}} K(2(n+1) \delta, .) *\left|u^{\delta}((p-2 n) \delta, .)-\mathcal{T}_{h}\left(u^{\delta}((p-2 n) \delta, .)\right)\right| \\
\quad \leq \int_{B_{R+L 2(n+1) \delta}} K(2(n+1) \delta, .) *\left|u^{\delta}((p-2 n-1) \delta, .)-\mathcal{T}_{h}\left(u^{\delta}((p-2 n-1) \delta, .)\right)\right| .
\end{aligned}
$$

Since

$$
\begin{aligned}
u^{\delta}((p-2 n-1) \delta, .)-\mathcal{T}_{h}\left(u^{\delta}\right. & ((p-2 n-1) \delta, .)) \\
& =K(2 \delta, .) *\left(u^{\delta}((p-2(n+1)) \delta, .)-\mathcal{T}_{h}\left(u^{\delta}((p-2(n+1)) \delta, .)\right)\right),
\end{aligned}
$$

the semi-group property of $K$ gives $I(n) \leq I(n+1)$, for all $0 \leq 2(n+1) \leq p$. Consequently, $I(0) \leq I(p / 2)$. Since $u^{\delta}$ satisfies the fractal equation on $\left.] p \delta,(p+1) \delta\right] \times \mathbb{R}^{N}$,

$$
\int_{B_{R}}\left|u^{\delta}((p+1) \delta, .)-\mathcal{T}_{h}\left(u^{\delta}((p+1) \delta, .)\right)\right| \leq I(0)
$$

and the proof of (5.2) is complete.

The rest of the proof is based on the following lemma.

Lemma 5.1. Let $E$ be a Banach space. Let $A \subset E$. If, for every $\mu>0$, there exists $A_{\mu} \subset E$ relatively compact such that $A \subseteq A_{\mu}+B(0, \mu)$, then $A$ is relatively compact in $E$.

This lemma is classical and for instance it is used in the proof of Kolmogorov's Theorem. Let $T>0$ and $R>0$. For all $\mu>0$, we define $u_{\mu}^{\delta}(t, x)=u^{\delta}(t,). * \rho_{\mu}(x)$, where $\rho_{\mu}$ is any approximate unit as before. Let us use the lemma above with $E=C\left([0, T] ; L^{1}\left(B_{R}\right)\right), A=\left\{u^{\delta}: \delta>0\right\}$ and $A_{\mu}=\left\{u_{\mu}^{\delta}: \delta>0\right\}$. Let us begin by the proof of the relative compactness of $A_{\mu}$ in $E$. By (5.1) and Young's Inequalities, $u_{\mu}^{\delta}(t,.) \in C_{b}^{\infty}\left(\mathbb{R}^{N}\right)$ and all its derivatives are bounded independently on $t$ and $\delta$. Moreover, the associativity of the convolution product implies that

$$
\left.\left.u_{\mu}^{\delta}(t, x)=K(2(t-\delta p), .) * u_{\mu}^{\delta}(p \delta, .)(x), \quad(t, x) \in\right] p \delta,(p+1) \delta\right] \times \mathbb{R}^{N},
$$

for all even $p$. Thus by Lemma $7.1, u_{\mu}^{\delta}$ is a classical solution to the fractal equation on $] p \delta,(p+$ 1) $\delta] \times \mathbb{R}^{N}$. By the continuity of $g$ from $W^{2, \infty}\left(\mathbb{R}^{N}\right)$ into $C_{b}\left(\mathbb{R}^{N}\right)$, the time derivative of $u_{\mu}^{\delta}$ is bounded, independently on $t$ and $\delta$, on each interval where the fractal equation is satisfied. Moreover, it is well-known that the entropy solution to the hyperbolic equation satisfies this equation in the distribution sense. In particular, $\partial_{t} u_{\mu}^{\delta}=-f\left(u^{\delta}\right) *_{x} \nabla \rho_{\mu}$ in $\mathcal{D}^{\prime}(] q \delta,(q+1) \delta\left[\times \mathbb{R}^{N}\right)$, for all odd $q$. Estimate (5.1) and Young's Inequalities thus give us a bound on the time derivative, independently on $t$ and $\delta$, on each interval where the hyperbolic equation is satisfied. To sum-up, we have proved that the family $A_{\mu}$ is bounded in $C_{b}\left([0, T] \times \bar{B}_{R}\right)$ and equilipschitz-continuous on $\left[0, \infty\left[\times \mathbb{R}^{N}\right.\right.$. By Ascoli-Arzela's Theorem, $A_{\mu}$ is relatively compact in $C_{b}\left([0, T] \times \bar{B}_{R}\right)$ and $a$ fortiori in $E$. Now, we define $\omega(\mu)=\sup _{\delta>0}\left\|u^{\delta}-u_{\mu}^{\delta}\right\|_{E}$. The set $A \subset A_{\mu}+B(0, \omega(\mu))$ and it suffices to prove that $\lim _{\mu \rightarrow 0} \omega(\mu)=0$ in order to use the lemma above. By (5.2), (5.3), Lemma 7.2 and the finite propagation speed for scalar conservation laws (to estimate the translations for all $t \geq 0)$,

$$
\limsup _{h \rightarrow 0} \sup _{\delta>0} \int_{t \in[0, T]}\left|u^{\delta}(t, .)-\mathcal{T}_{h}\left(u^{\delta}(t, .)\right)\right|=0
$$

and classically with approximate units, $\lim _{\mu \rightarrow 0} \omega(\mu)=0$. The proof of the relative compactness of $A$ in $E$ and thus of $\left\{u^{\delta}: \delta>0\right\}$ in $C\left([0, T] ; L_{l o c}^{1}\left(\mathbb{R}^{N}\right)\right.$ ) (for all $\left.T>0\right)$ is now complete. 


\subsection{Entropy inequality for $u^{\delta}$}

Recalling that $u_{\mu}^{\delta}$ is a classical solution to the fractal equation (thus of (1.1) for $f=0$ and $g$ replaced by $2 g$ ), it is already proved in Section 2 (see the proof of Proposition 2.1 i)) that

$$
\begin{aligned}
& \int_{p \delta}^{(p+1) \delta} \int_{\mathbb{R}^{N}} \eta\left(u_{\mu}^{\delta}\right) \partial_{t} \varphi \\
&+2 c_{N}(\lambda) \int_{p \delta}^{(p+1) \delta} \int_{\mathbb{R}^{N}} \int_{|z| \geq r} \eta^{\prime}\left(u_{\mu}^{\delta}(t, x)\right) \frac{u_{\mu}^{\delta}(t, x+z)-u_{\mu}^{\delta}(t, x)}{|z|^{N+\lambda}} \varphi(t, x) d z d x d t \\
&+2 c_{N}(\lambda) \int_{p \delta}^{(p+1) \delta} \int_{\mathbb{R}^{N}} \int_{|z| \leq r} \eta\left(u_{\mu}^{\delta}(t, x)\right) \frac{\varphi(t, x+z)-\varphi(x)-\nabla \varphi(t, x) . z}{|z|^{N+\lambda}} d z d x d t \\
& \quad+\int_{\mathbb{R}^{N}} \eta\left(u_{\mu}^{\delta}(p \delta, .)\right) \varphi(p \delta, .)-\int_{\mathbb{R}^{N}} \eta\left(u_{\mu}^{\delta}((p+1) \delta, .)\right) \varphi((p+1) \delta, .) \geq 0
\end{aligned}
$$

for all $r>0$, all smooth entropy $\eta$ and all non-negative $\varphi \in C_{c}^{\infty}\left(\left[0, \infty\left[\times \mathbb{R}^{N}\right)\right.\right.$ (note that $\varphi$ is not necessarily null for $t=(p+1) \delta$ and we get a new boundary term). Moreover, $u_{\mu}^{\delta}$ converges to $u^{\delta}$ as $\mu \rightarrow 0$ a.e. on $] p \delta,(p+1) \delta\left[\times \mathbb{R}^{N}\right.$ and by $(5.1), u_{\mu}^{\delta}$ stays bounded by $\left\|u_{0}\right\|_{L^{\infty}\left(\mathbb{R}^{N}\right)}$. The limit $\mu \rightarrow 0$ in (5.5), thanks to the continuity of $\eta$ and $\eta^{\prime}$ and to the dominated convergence theorem, then gives

$$
\begin{aligned}
\int_{p \delta}^{(p+1) \delta} & \int_{\mathbb{R}^{N}} \eta\left(u^{\delta}\right) \partial_{t} \varphi \\
& +2 c_{N}(\lambda) \int_{p \delta}^{(p+1) \delta} \int_{\mathbb{R}^{N}} \int_{|z| \geq r} \eta^{\prime}\left(u^{\delta}(t, x)\right) \frac{u^{\delta}(t, x+z)-u^{\delta}(t, x)}{|z|^{N+\lambda}} \varphi(t, x) d z d x d t \\
+ & 2 c_{N}(\lambda) \int_{p \delta}^{(p+1) \delta} \int_{\mathbb{R}^{N}} \int_{|z| \leq r} \eta\left(u^{\delta}(t, x)\right) \frac{\varphi(t, x+z)-\varphi(x)-\nabla \varphi(t, x) . z}{|z|^{N+\lambda}} d z d x d t \\
& +\int_{\mathbb{R}^{N}} \eta\left(u^{\delta}(p \delta, .)\right) \varphi(p \delta, .)-\int_{\mathbb{R}^{N}} \eta\left(u^{\delta}((p+1) \delta, .)\right) \varphi((p+1) \delta, .) \geq 0,
\end{aligned}
$$

for all even $p$. Consider now any flux $\phi$ associated to $\eta$. Let us recall that, as far as the hyperbolic equation is concerned, the following inequality is taken as definition of entropy solution. ${ }^{2}$

$$
\begin{aligned}
\int_{q \delta}^{(q+1) \delta} \int_{\mathbb{R}^{N}} \eta\left(u^{\delta}\right) \partial_{t} \varphi & +2 \int_{q \delta}^{(q+1) \delta} \int_{\mathbb{R}^{N}} \phi\left(u^{\delta}\right) \nabla \varphi \\
& +\int_{\mathbb{R}^{N}} \eta\left(u^{\delta}(q \delta, .)\right) \varphi(q \delta, .)-\int_{\mathbb{R}^{N}} \eta\left(u^{\delta}((q+1) \delta, .)\right) \varphi((q+1) \delta, .) \geq 0,
\end{aligned}
$$

for all odd $q$. Summing now (5.6) and (5.7) for all even $p$ and all odd $q$ (notice that since $\varphi(t,)=$.0 for $t$ sufficiently large, these sums are finite and we keep only the boundary term for

\footnotetext{
${ }^{2}$ The presence of the boundary term for $t=(q+1) \delta$, that generally not appears in the definition, is obtained by the continuity with values in $L_{l o c}^{1}\left(\mathbb{R}^{N}\right)$ of the solution.
} 
$t=0)$,

$$
\begin{aligned}
& \int_{0}^{\infty} \int_{\mathbb{R}^{N}} \eta\left(u^{\delta}(t, x)\right) \partial_{t} \varphi(t, x)+\phi\left(u^{\delta}(t, x)\right) \nabla \varphi(t, x) 2\left(1-\chi_{\delta}(t)\right) d x d t \\
& \quad+c_{N}(\lambda) \int_{0}^{\infty} \int_{\mathbb{R}^{N}} \int_{|z| \geq r} \eta^{\prime}\left(u^{\delta}(t, x)\right) \frac{u^{\delta}(t, x+z)-u^{\delta}(t, x)}{|z|^{N+\lambda}} \varphi(t, x) 2 \chi_{\delta}(t) d z d x d t \\
& \quad+c_{N}(\lambda) \int_{0}^{\infty} \int_{\mathbb{R}^{N}} \int_{|z| \leq r} \eta\left(u^{\delta}(t, x)\right) \frac{\varphi(t, x+z)-\varphi(t, x)-\nabla \varphi(t, x) \cdot z}{|z|^{N+\lambda}} 2 \chi_{\delta}(t) d z d x d t \\
& \left.+\int_{\mathbb{R}^{N}} \eta\left(u_{0}\right)\right) \varphi(0, .) \geq 0
\end{aligned}
$$

where $\chi_{\delta}:=\sum_{\text {even } p} \mathbf{1}_{] p \delta,(p+1) \delta]}$.

\subsection{Passing to the limit as $\delta \rightarrow 0$}

Since $u^{\delta}$ converges as $\delta \rightarrow 0$ to $u$ in $C\left([0, T] ; L_{\text {loc }}^{1}\left(\mathbb{R}^{N}\right)\right.$ ) (for all $T>0$ ), up to a subsequence, the convergence still holds true in $L_{l o c}^{1}(] 0, \infty\left[\times \mathbb{R}^{N}\right)$ and a fortiori a.e. on $] 0, \infty\left[\times \mathbb{R}^{N}\right.$, up to a subsequence. Using again (5.1) and the continuity of $\eta^{\prime}$, the dominated convergence theorem implies that

$$
\begin{aligned}
\int_{\mathbb{R}^{N}} \int_{|z| \geq r} \eta^{\prime}\left(u^{\delta}(., x)\right) & \frac{u^{\delta}(., x+z)-u^{\delta}(., x)}{|z|^{N+\lambda}} \varphi(., x) d z d x \\
& \rightarrow \int_{\mathbb{R}^{N}} \int_{|z| \geq r} \eta^{\prime}(u(., x)) \frac{u(., x+z)-u(., x)}{|z|^{N+\lambda}} \varphi(., x) d z d x \quad \text { in } L^{1}(] 0, \infty[),
\end{aligned}
$$

as $\delta \rightarrow 0$. Classically $2 \chi_{\delta} \rightarrow 1$ in $L^{\infty}(] 0, \infty[)$ weak-* and the second term of (5.8) converges to the second term of $(2.2)$, as $\delta \rightarrow 0$. Similar arguments allow to pass to the limit in the other terms and deduce that $u$ is an entropy solution to (1.1). The proof of Theorem 3.1 is now complete.

Remark 5.1. Since the solutions to both the hyperbolic and the fractal equations satisfy the properties of Proposition 3.1, it is quite obvious that $u^{\delta}$ also satisfies these properties and thus so does $u$.

\section{Vanishing viscosity method}

We conclude this work by the proof of the convergence results of Theorem 3.3.

\subsection{Error estimates}

We begin with the case $u_{0} \in L^{\infty}\left(\mathbb{R}^{N}\right) \cap L^{1}\left(\mathbb{R}^{N}\right) \cap B V\left(\mathbb{R}^{N}\right)$. We use again the doubling variable technique, as in [12] for $\lambda=2$ and in [6] for $\lambda \in] 1,2]$. In the sequel, we let $C$ denote any non-negative constant that only depends on $T, N, \lambda$ and the $L^{1} \cap B V$ norm of $u_{0}$. Let us recall that by definition,

$$
\int_{0}^{\infty} \int_{\mathbb{R}^{N}}\left(\eta(u) \partial_{t} \varphi+\phi(u) . \nabla \varphi\right)+\int_{\mathbb{R}^{N}} \eta\left(u_{0}\right) \varphi(0, .) \geq 0
$$


for all entropy-flux pair $(\eta, \phi)$ and all non-negative $\varphi \in C_{c}^{\infty}\left(\left[0, \infty\left[\times \mathbb{R}^{N}\right)\right.\right.$. Arguing as in Subsection 4.1 , we get

$$
\begin{gathered}
\begin{array}{l}
\int_{0}^{\infty} \int_{0}^{\infty} \int_{\mathbb{R}^{N}} \int_{\mathbb{R}^{N}}\left|u^{\varepsilon}(t, x)-u(s, y)\right|\left(\partial_{t}+\partial_{s}\right) \psi(t, s, x, y) \\
+F\left(u^{\varepsilon}(t, x), u(s, y)\right) \cdot\left(\nabla_{x}+\nabla_{y}\right) \psi(t, s, x, y) d y d x d s d t
\end{array} \\
+C \varepsilon \int_{0}^{\infty} \int_{0}^{\infty} \int_{\mathbb{R}^{N}} \int_{\mathbb{R}^{N}} \int_{|z| \geq r} \psi(t, s, x, y) \\
\begin{array}{r}
\operatorname{sign}\left(u^{\varepsilon}(t, x)-u(s, y)\right) \frac{u^{\varepsilon}(t, x+z)-u^{\varepsilon}(t, x)}{|z|^{N+\lambda}} d z d y d x d s d t \\
+C \varepsilon \int_{0}^{\infty} \int_{0}^{\infty} \int_{\mathbb{R}^{N}} \int_{\mathbb{R}^{N}} \int_{|z| \leq r}\left|u^{\varepsilon}(t, x)-u(s, y)\right| \\
+\int_{0}^{\infty} \int_{\mathbb{R}^{N}} \int_{\mathbb{R}^{N}}\left|u_{0}(x)-u(s, y)\right| \psi(0, s, x, y) d y d x d s \\
+\int_{0}^{\infty} \int_{\mathbb{R}^{N}} \int_{\mathbb{R}^{N}}\left|u^{\varepsilon}(t, x)-u_{0}(y)\right| \psi(t, 0, x, y) d y d x d t \geq 0 .
\end{array}
\end{gathered}
$$

We take $\psi(t, s, x, y)=\theta_{\nu}(s-t) \rho_{\mu}(y-x) \Theta(t)$. In fact, $u^{\varepsilon}$ and $u$ are in $L^{1}$ and there is no integrability problem. Note that, as in Subsection $4.2, \Theta$ will be chosen as a primitive of an approximate unit and we still let $C$ denote some constants also depending on $\int_{0}^{\infty} \Theta \leq T, \int_{0}^{\infty}\left|\Theta^{\prime}\right|=1$ and $\Theta(0)=1$. We have

$$
\begin{aligned}
& \int_{0}^{\infty} \int_{0}^{\infty} \int_{\mathbb{R}^{N}} \int_{\mathbb{R}^{N}}\left|u^{\varepsilon}(t, x)-u(s, y)\right| \theta_{\nu}(s-t) \rho_{\mu}(y-x) \Theta^{\prime}(t) d y d x d s d t \\
& +C \varepsilon \int_{0}^{\infty} \int_{0}^{\infty} \int_{\mathbb{R}^{N}} \int_{\mathbb{R}^{N}} \int_{|z| \geq r} \theta_{\nu}(s-t) \rho_{\mu}(y-x) \Theta(t) \\
& \operatorname{sign}\left(u^{\varepsilon}(t, x)-u(s, y)\right) \frac{u^{\varepsilon}(t, x+z)-u^{\varepsilon}(t, x)}{|z|^{N+\lambda}} d z d y d x d s d t \\
& +C \varepsilon \int_{0}^{\infty} \int_{0}^{\infty} \int_{\mathbb{R}^{N}} \int_{\mathbb{R}^{N}} \int_{|z| \leq r}\left|u^{\varepsilon}(t, x)-u(s, y)\right| \theta_{\nu}(s-t) \Theta(t) \\
& \frac{\rho_{\mu}(y-x-z)-\rho_{\mu}(y-x)+\nabla \rho_{\mu}(y-x) . z}{|z|^{N+\lambda}} d z d y d x d s d t \\
& +\int_{0}^{\infty} \int_{\mathbb{R}^{N}} \int_{\mathbb{R}^{N}}\left|u_{0}(x)-u(s, y)\right| \theta_{\nu}(s) \rho_{\mu}(y-x) \Theta(0) d y d x d s \geq 0 .
\end{aligned}
$$

We let $I_{r}$ and $J_{r}$ denote respectively the second and the third term of (6.1). We have

$$
\begin{aligned}
I_{r} & \leq C \varepsilon \int_{0}^{\infty} \int_{0}^{\infty} \int_{\mathbb{R}^{N}} \int_{\mathbb{R}^{N}} \int_{|z| \geq r} \theta_{\nu}(s-t) \rho_{\mu}(y-x) \Theta(t) \\
& =C \varepsilon \int_{0}^{\infty} \int_{\mathbb{R}^{N}} \int_{|z| \geq r} \Theta(t) \frac{\left|u^{\varepsilon}(t, x+z)-u^{\varepsilon}(t, x)\right|}{|z|^{N+\lambda}} d z d y d x d s d t,
\end{aligned}
$$


Integrating first w.r.t. $x$,

$$
I_{r} \leq C \varepsilon \int_{0}^{\infty} \int_{|z| \geq r} \Theta(t)|z|^{-N-\lambda}\left\|\mathcal{T}_{-z}\left(u^{\varepsilon}(t, .)\right)-u^{\varepsilon}(t, .)\right\|_{L^{1}\left(\mathbb{R}^{N}\right)} d z d t
$$

By Proposition 3.1 iii) and iv),

$$
\begin{aligned}
& \left\|\mathcal{T}_{-z}\left(u^{\varepsilon}(t, .)\right)-u^{\varepsilon}(t, .)\right\|_{L^{1}\left(\mathbb{R}^{N}\right)} \leq 2|| u_{0} \|_{L^{1}\left(\mathbb{R}^{N}\right)} \\
& \quad \text { and }\left\|\mathcal{T}_{-z}\left(u^{\varepsilon}(t, .)\right)-\left.u^{\varepsilon}(t, .)\right|_{L^{1}\left(\mathbb{R}^{N}\right)} \leq\right\| u^{\varepsilon}(t, .) \|\left.\right|_{B V\left(\mathbb{R}^{N}\right)}|z| \leq\left|u_{0}\right|_{B V\left(\mathbb{R}^{N}\right)}|z|,
\end{aligned}
$$

for all $t>0$. For $0<r \leq 1$, let us cut the integral term of (6.2) in two pieces according as $|z| \geq 1$ or not. Let us use both last estimates on each of one part, respectively. We get

$$
\begin{aligned}
I_{r} \leq C \varepsilon \int_{0}^{\infty} \int_{|z| \geq 1} \Theta(t)|z|^{-N-\lambda} d z d t & \\
& +C \varepsilon \int_{0}^{\infty} \int_{r \leq|z| \leq 1} \Theta(t)|z|^{-N-\lambda+1} d z d t \leq C \varepsilon+C \varepsilon \int_{r}^{1} \frac{d \tau}{\tau^{\lambda}} .
\end{aligned}
$$

Let us now bound from above $J_{r}$. By Taylor's Formula and Fubini's Theorem,

$$
\begin{gathered}
J_{r}=C \varepsilon \int_{0}^{1} \int_{0}^{\infty} \int_{0}^{\infty} \int_{\mathbb{R}^{N}} \int_{\mathbb{R}^{N}} \int_{|z| \leq r}\left|u^{\varepsilon}(t, x)-u(s, y)\right|(1-\tau) \theta_{\nu}(s-t) \Theta(t) \\
=C \varepsilon \int_{0}^{1} \int_{0}^{\infty} \int_{0}^{\infty} \int_{\mathbb{R}^{N}} \int_{|z| \leq r}(1-\tau) \theta_{\nu}(s-t) \Theta(t)|z|^{-N-\lambda} \\
\left.\left(\int_{\mathbb{R}^{N}}\left|u^{\varepsilon}(t, x)-u(s, y)\right| D^{2} \rho_{\mu}(y-x-\tau-\tau) z \cdot z\right) z . z d x\right) d z d y d s d t d \tau .
\end{gathered}
$$

By Lemma $7.3,\left|u^{\varepsilon}(t,)-.u(s, y)\right| \in B V\left(\mathbb{R}^{N}\right)$ and an integration by parts gives

$$
\begin{aligned}
& J_{r}=C \varepsilon \int_{0}^{1} \int_{0}^{\infty} \int_{0}^{\infty} \int_{\mathbb{R}^{N}} \int_{|z| \leq r}(1-\tau) \theta_{\nu}(s-t) \Theta(t)|z|^{-N-\lambda} \\
&\left(\int_{\mathbb{R}^{N}} \nabla \rho_{\mu}(y-x-\tau z) . z d\left(D\left(\left|u^{\varepsilon}(t, .)-u(s, y)\right|\right)\right)(x) . z\right) d z d y d s d t d \tau,
\end{aligned}
$$

where the integral is taken w.r.t. the bounded borel measure $D\left(\left|u^{\varepsilon}(t,)-.u(s, y)\right|\right) . z$. By Fubini's Theorem,

$$
\begin{aligned}
J_{r}=C \varepsilon \int_{0}^{1} \int_{0}^{\infty} \int_{0}^{\infty} \int_{|z| \leq r}(1-\tau) \theta_{\nu}(s-t) \Theta(t)|z|^{-N-\lambda} \\
\\
\quad\left(\int_{\mathbb{R}^{N}}\left(\int_{\mathbb{R}^{N}} \nabla \rho_{\mu}(y-x-\tau z) . z d\left(D\left(\left|u^{\varepsilon}(t, .)-u(s, y)\right|\right)\right)(x) . z\right) d y\right) d z d s d t d \tau .
\end{aligned}
$$

By Lemma 7.3, $\left|D\left(\left|u^{\varepsilon}(t,)-.u(s, y)\right|\right)\right| \leq\left|D u^{\varepsilon}(t,).\right|$ and the term in parenthesis above is lower than $|z|^{2} \int_{\mathbb{R}^{N}}\left(\int_{\mathbb{R}^{N}}\left|\nabla \rho_{\mu}(y-x-\tau z)\right| d\left(\left|D u^{\varepsilon}(t,).\right|\right)(x)\right) d y$. Note that $\left|D u^{\varepsilon}(t,).\right|$ is finite and its 
tensor product by the Lebesgue measure satisfies the hypothesis of Fubini's theorem. Then, this integral is well-defined and we can moreover first integrate w.r.t. $y$ to bound it from above by

$$
|z|^{2}\left|u^{\varepsilon}(t, .)\right|_{B V\left(\mathbb{R}^{N}\right)}|| \nabla \rho_{\mu}\left\|_{\left(L^{1}\left(\mathbb{R}^{N}\right)\right)^{N}} \leq|z|^{2}\left|u_{0}\right|_{B V\left(\mathbb{R}^{N}\right)}|| \nabla \rho_{\mu}\right\|_{\left(L^{1}\left(\mathbb{R}^{N}\right)\right)^{N}}
$$

With smoothing kernel of the form $\rho_{\mu}=\mu^{-N} \rho\left(\mu^{-1}\right.$.), we have $\left\|\nabla \rho_{\mu}\right\|_{L^{1}\left(\mathbb{R}^{N}\right)} \leq C \mu^{-1}$. Then

$$
\begin{aligned}
J_{r} & \leq C \varepsilon \mu^{-1} \int_{0}^{1} \int_{0}^{\infty} \int_{0}^{\infty} \int_{|z| \leq r}(1-\tau) \theta_{\nu}(s-t) \Theta(t)|z|^{-N+2-\lambda} d z d s d t d \tau \\
& =C \varepsilon \mu^{-1} r^{2-\lambda} .
\end{aligned}
$$

Using (6.1) and (6.3),

$$
\begin{gathered}
\int_{0}^{\infty} \int_{0}^{\infty} \int_{\mathbb{R}^{N}} \int_{\mathbb{R}^{N}}\left|u^{\varepsilon}(t, x)-u(s, y)\right| \theta_{\nu}(s-t) \rho_{\mu}(y-x) \Theta^{\prime}(t) d y d x d s d t \\
+\int_{0}^{\infty} \int_{\mathbb{R}^{N}} \int_{\mathbb{R}^{N}}\left|u_{0}(x)-u(s, y)\right| \theta_{\nu}(s) \rho_{\mu}(y-x) \Theta(0) d y d x d s \\
\geq-C \varepsilon-C \varepsilon \int_{r}^{1} \frac{d \tau}{\tau^{\lambda}}-C \varepsilon \mu^{-1} r^{2-\lambda}
\end{gathered}
$$

Recalling (4.7) and (4.9) derived in Subsection 4.1,

$$
\int_{0}^{\infty} \int_{\mathbb{R}^{N}}\left|u^{\varepsilon}-u\right| \Theta^{\prime} \geq-\omega_{1}(\nu, \mu)-\omega_{2}(\mu)-\omega_{3}(\nu, \mu)-C \varepsilon-C \varepsilon \int_{r}^{1} \frac{d \tau}{\tau^{\lambda}}-C \varepsilon \mu^{-1} r^{2-\lambda},
$$

where the role of $u$ and $v$ are now played by $u^{\varepsilon}$ and $u$, respectively. By Fubini's Theorem,

$$
\omega_{1}(\nu, \mu) \leq \int_{0}^{\infty} \theta_{\nu}\left(s^{\prime}\right)\left(\int_{\mathbb{R}^{N}}\left\|\left(u-\mathcal{T}_{\left(-s^{\prime},-y^{\prime}\right)} u\right) \Theta^{\prime}\right\|_{L^{1}(] 0, \infty\left[\times \mathbb{R}^{N}\right)} \rho_{\mu}\left(y^{\prime}\right) d y^{\prime}\right) d s^{\prime} .
$$

Since $u \in L^{\infty}(] 0, \infty\left[; L^{1}\left(\mathbb{R}^{N}\right)\right)$ and $\Theta^{\prime} \in C_{c}^{\infty}\left(\left[0, \infty[)\right.\right.$, the term $\left(\mathcal{T}_{\left(-s^{\prime},-y^{\prime}\right)} u\right) \Theta^{\prime}$ is continuous w.r.t. $\left(s^{\prime}, y^{\prime}\right)$ from $\left[0, \infty\left[\times \mathbb{R}^{N}\right.\right.$ into $L^{1}(] 0, \infty\left[\times \mathbb{R}^{N}\right)$. Since $\rho_{\mu} \in C_{c}^{\infty}\left(\mathbb{R}^{N}\right)$,

$$
s^{\prime} \in\left[0, \infty\left[\rightarrow \int_{\mathbb{R}^{N}}\left\|\left(u-\mathcal{T}_{\left(-s^{\prime},-y^{\prime}\right)} u\right) \Theta^{\prime}\right\|_{L^{1}(] 0, \infty\left[\times \mathbb{R}^{N}\right)} \rho_{\mu}\left(y^{\prime}\right) d y^{\prime}\right.\right.
$$

is continuous. Consequently,

$$
\begin{aligned}
\underset{\nu \rightarrow 0}{\limsup \omega_{1}(\nu, \mu)} & \leq \int_{\mathbb{R}^{N}}\left\|\left(u-\mathcal{T}_{\left(0,-y^{\prime}\right)} u\right) \Theta^{\prime}\right\|_{L^{1}(] 0, \infty\left[\times \mathbb{R}^{N}\right)} \rho_{\mu}\left(y^{\prime}\right) d y^{\prime} \\
& =\int_{0}^{\infty} \int_{\mathbb{R}^{N}}\left\|u(t, .)-\mathcal{T}_{-y^{\prime}}(u(t, .))\right\|_{L^{1}\left(\mathbb{R}^{N}\right)} \Theta^{\prime}(t) \rho_{\mu}\left(y^{\prime}\right) d y^{\prime} d t \\
& \leq C \int_{0}^{\infty} \int_{\mathbb{R}^{N}}\left|\Theta^{\prime}(t) \| y^{\prime}\right| \rho_{\mu}\left(y^{\prime}\right) d y^{\prime} d t \\
& \leq C \mu
\end{aligned}
$$

since $\operatorname{supp}\left(\rho_{\mu}\right) \subset B_{\mu}$ and the $B V$ semi norm of $u$ is non-increased. Moreover, $\omega_{2}(\mu) \leq C \mu$ and recalling that $\lim _{\nu \rightarrow 0} \omega_{3}(\nu, \mu)=0$ (for all $\mu>0$ ), the limit $\nu \rightarrow 0$ in (6.5) gives

$$
\int_{0}^{\infty} \int_{\mathbb{R}^{N}}\left|u-u^{\varepsilon}\right| \Theta^{\prime} \geq-C \mu-C \varepsilon-C \varepsilon \int_{r}^{1} \frac{d \tau}{\tau^{\lambda}}-C \varepsilon \mu^{-1} r^{2-\lambda} .
$$


We take $\Theta$ as in Remark 4.1 of Subsection 4.2. Then

$$
\int_{0}^{\infty}\left(\int_{\mathbb{R}^{N}}\left|u(t, .)-u^{\varepsilon}(t, .)\right|\right) \theta_{\nu}(T-t) d t \leq C \mu+C \varepsilon+C \varepsilon \int_{r}^{1} \frac{d \tau}{\tau^{\lambda}}+C \varepsilon \mu^{-1} r^{2-\lambda}
$$

and letting $\nu \rightarrow 0, \int_{\mathbb{R}^{N}}\left|u(T,)-.u^{\varepsilon}(T,).\right| \leq C \mu+C \varepsilon+C \varepsilon \int_{r}^{1} \frac{d \tau}{\tau^{\lambda}}+C \varepsilon \mu^{-1} r^{2-\lambda}$. The limits in order $r \rightarrow 0$ and $\mu \rightarrow 0$, for $\lambda \in] 0,1$ [, and an optimization w.r.t. $r$ and $\mu$ (which gives $r=\mu=\varepsilon$ ), for $\lambda=1$, then give the error estimates of Theorem 3.3.

\subsection{Convergence for $u_{0}$ only $L^{\infty}$}

Let us approximate $u_{0}$ in $L_{\text {loc }}^{1}\left(\mathbb{R}^{N}\right)$ by $u_{0}^{n} \in L^{\infty}\left(\mathbb{R}^{N}\right) \cap L^{1}\left(\mathbb{R}^{N}\right) \cap B V\left(\mathbb{R}^{N}\right)$ bounded by $\left\|u_{0}\right\|_{L^{\infty}\left(\mathbb{R}^{N}\right)}$. Recalling that $K(\varepsilon .,$.$) is the kernel of the fractal operator \varepsilon g$, the finite propagation speed and the finite-infinite propagation speed results imply that for all $T, R>0$,

$$
\begin{aligned}
& \left\|u^{\varepsilon}-u\right\|_{C\left([0, T] ; L^{1}\left(B_{R}\right)\right)} \\
& \leq\left\|u^{\varepsilon}-u_{n}^{\varepsilon}\right\|_{C\left([0, T] ; L^{1}\left(B_{R}\right)\right)}+\left\|u_{n}^{\varepsilon}-u_{n}\right\|_{C\left([0, T] ; L^{1}\left(B_{R}\right)\right)}+\left\|u_{n}-u\right\|_{C\left([0, T] ; L^{1}\left(B_{R}\right)\right)}, \\
& \quad \leq \sup _{t \in[0, T]} \int_{B_{R+L t}} K(\varepsilon t, .) *\left|u_{0}-u_{0}^{n}\right|+C_{n} \omega(\varepsilon)+\left\|u_{0}^{n}-u_{0}\right\|_{L^{1}\left(B_{R+L T}\right)},
\end{aligned}
$$

where $C_{n}$ and $\omega(\varepsilon)$ derive from the last Subsection. We have proved that $C_{n}$ does not depend on $\varepsilon$ and that $\lim _{\varepsilon \rightarrow 0} \omega(\varepsilon)=0$. By (7.3),

$$
\limsup _{\varepsilon \rightarrow 0} \sup _{t \in[0, T]} \int_{B_{R+L t}} K(\varepsilon t, .) *\left|u_{0}-u_{0}^{n}\right| \leq\left\|u_{0}-u_{0}^{n}\right\|_{L^{1}\left(B_{R+L T+r}\right)},
$$

for all $r>0$. The limit $r \rightarrow 0$ then gives

$$
\limsup _{\varepsilon \rightarrow 0} \sup _{t \in[0, T]} \int_{B_{R+L t}} K(\varepsilon t, .) *\left|u_{0}-u_{0}^{n}\right| \leq\left\|u_{0}-u_{0}^{n}\right\|_{L^{1}\left(B_{R+L T}\right)} .
$$

Recalling (6.6), $\lim \sup _{\varepsilon \rightarrow 0}\left\|u^{\varepsilon}-u\right\|_{C\left([0, T] ; L^{1}\left(B_{R}\right)\right)} \leq 2\left\|u_{0}-u_{0}^{n}\right\|_{L^{1}\left(B_{R+L T}\right)}$ and the limit $n \rightarrow+\infty$ completes the proof.

\section{Appendix}

\subsection{Technical results}

Lemma 7.1. Let $T>0$ and $\varphi \in C_{c}^{\infty}\left([0, T] \times \mathbb{R}^{N}\right)$. Define $w=K *_{x} \varphi$. Then, $w \in C_{b}^{\infty}([0, T] \times$ $\left.\mathbb{R}^{N}\right)$ and

$$
\partial_{t} w(t, x)-K(t, .) * \partial_{t} \varphi(t, .)(x)+g[w(t, .)](x)=0, \quad 0<t \leq T, x \in \mathbb{R}^{N} .
$$

Let us now assume that $\varphi$ does not depend on $t$ and only belongs to $C_{b}^{\infty}\left(\mathbb{R}^{N}\right)$. Then $w \in$ $C_{b}^{\infty}\left(\left[0, \infty\left[\times \mathbb{R}^{N}\right)\right.\right.$ and $w$ is a classical solution to the fractal equation $\partial_{t} w+g[w]=0$ on $] 0, \infty\left[\times \mathbb{R}^{N}\right.$. 
Proof of Lemma 7.1. Let $T>0$ and $\varphi \in C_{c}^{\infty}\left([0, T] \times \mathbb{R}^{N}\right)$. By Fourier transform,

$$
w(t, x)=\mathcal{F}^{-1}\left(e^{-t|\cdot|^{\lambda}}\right) * \varphi(t, .)(x)=\mathcal{F}^{-1}\left(e^{-t|\cdot|^{\lambda}} \mathcal{F}(\varphi(t, .))\right)(x) .
$$

By the theorem of derivation under the integral sign, we immediately get that $w$ is smooth with all its derivatives bounded. Moreover,

$$
\begin{aligned}
\partial_{t} w(t, x) & =-\mathcal{F}^{-1}\left(|.|^{\lambda} e^{-t|\cdot|^{\lambda}} \mathcal{F}(\varphi(t, .))\right)(x)+\mathcal{F}^{-1}\left(e^{-t|\cdot|^{\lambda}} \mathcal{F}\left(\partial_{t} \varphi(t, .)\right)\right)(x), \\
& =-B+K(t, .) * \partial_{t} \varphi(t, .)(x),
\end{aligned}
$$

where $B=\mathcal{F}^{-1}\left(e^{-t|\cdot|^{\lambda}}\left(|\cdot|^{\lambda} \mathcal{F}(\varphi(t,)).\right)\right)(x)$. Next,

$$
B=K(t, .) * \mathcal{F}^{-1}\left(|.|^{\lambda} \mathcal{F}(\varphi(t, .))\right)(x)=K(t, .) * g[\varphi(t, .)](x) .
$$

Since $\varphi(t,.) \in \mathcal{S}\left(\mathbb{R}^{N}\right)$, Theorem 2.1 can be applied. By Fubini's Theorem, we get

$$
\begin{aligned}
& B=-c_{N}(\lambda) \int_{\mathbb{R}^{N}} \int_{|z| \geq r} K(t, y) \frac{\varphi(t, x-y+z)-\varphi(t, x-y)}{|z|^{N+\lambda}} d z d y \\
& -c_{N}(\lambda) \int_{\mathbb{R}^{N}} \int_{|z| \leq r} K(t, y) \frac{\varphi(t, x-y+z)-\varphi(t, x-y)-\nabla \varphi(t, x-y) \cdot z}{|z|^{N+\lambda}} d z d y, \\
& =-c_{N}(\lambda) \int_{|z| \geq r} \frac{w(t, x+z)-w(t, x)}{|z|^{N+\lambda}} d z \\
& \quad-c_{N}(\lambda) \int_{|z| \leq r} \frac{w(t, x+z)-w(t, x)-K(t, .) * \nabla \varphi(t, .)(x) . z}{|z|^{N+\lambda}} d z,
\end{aligned}
$$

where we have first integrated w.r.t. $y$ to get the last equality. We complete the proof of Lemma 7.1 for $\varphi \in C_{c}^{\infty}\left([0, T] \times \mathbb{R}^{N}\right)$ by using $\nabla w(t, x)=K(t,). * \nabla \varphi(t,).(x)$.

Let us now assume that $\varphi$ does not depend on $t$ and only belongs to $C_{b}^{\infty}\left(\mathbb{R}^{N}\right)$. Consider $\varphi_{n} \in C_{c}^{\infty}\left(\mathbb{R}^{N}\right)$ such that all its derivatives converge to all the derivatives of $\varphi$ locally uniformly on $\mathbb{R}^{N}$ and stay bounded independently on $n$. Define $w_{n}(t, x)=K(t,). * \varphi_{n}(x)$. By the preceding step, $w_{n}$ is a classical solution to the fractal equation. By the homogeneity property of $K$,

$$
\sup _{t \in[0, T]} \int_{\mathbb{R}^{N} \backslash B_{r}} K(t, .) \leq \int_{\mathbb{R}^{N} \backslash B_{T^{-1 / \lambda_{r}}}} K(1, .),
$$

for all $T>0$ and all $r>0$ and the family $\{K(t,):. 0<t \leq T\}$ is equi-integrable at the infinity. By similar cutting of approximate units as in the proof of the next lemma, one can see that all the spacial derivatives of $w_{n}$ converge locally uniformly on $\left[0, \infty\left[\times \mathbb{R}^{N}\right.\right.$ to all the derivatives of $w$ and stay bounded independently on $n$. By (2.1), so do the fractional (spatial) derivatives (see [8, Proposition 2.1] for more details) and by the fractal equation, so do the first temporal derivative. Moreover, the theorem of the derivation under the integral sign implies that all the derivatives of $w_{n}$ satisfy the fractal equation. Arguing by induction, all the derivatives (classical and fractional) of $w_{n}$ converge to all the derivatives of $w$ locally uniformly on $\left[0, \infty\left[\times \mathbb{R}^{N}\right.\right.$ and stay bounded independently on $n$. In particular, $w \in C_{b}^{\infty}\left(\left[0, \infty\left[\times \mathbb{R}^{N}\right)\right.\right.$ and the limit in the fractal equation completes the proof. 
Lemma 7.2. Let $w \in L^{\infty}\left(\mathbb{R}^{N}\right)$. Then for all $L, R, T, r, \varepsilon>0$,

$$
\begin{aligned}
\sup _{t \in[0, T]} \int_{B_{R+L t}} K(\varepsilon t, .) *|w| & \\
& \leq\|w\|_{L^{1}\left(B_{R+L T+r}\right)}+\|w\|_{L^{\infty}\left(\mathbb{R}^{N}\right)} \operatorname{mes}\left(B_{R+L T}\right) \int_{\mathbb{R}^{N} \backslash B_{(\varepsilon T)^{-1 / \lambda}}} K(1, .) .
\end{aligned}
$$

Proof of Lemma 7.2. Still using (7.2),

$$
\begin{aligned}
& \int_{B_{R}} K(\varepsilon t, .) *|w| \\
& =\int_{B_{r}} K(\varepsilon t, y)\left(\int_{B_{R+L t}}|w(x-y)| d x\right) d y \\
& \quad+\int_{\mathbb{R}^{N} \backslash B_{r}} K(\varepsilon t, y)\left(\int_{B_{R+L t}}|w(x-y)| d x\right) d y, \\
& \leq\|w\|_{L^{1}\left(B_{R+L t+r}\right)}+\|w\|_{L^{\infty}\left(\mathbb{R}^{N}\right)} \operatorname{mes}\left(B_{R+L t}\right) \int_{\mathbb{R}^{N} \backslash B_{(\varepsilon T)^{-1 / \lambda_{r}}}} K(1, .) .
\end{aligned}
$$

Let us then take the supremum w.r.t. $t \in[0, T]$ to complete the proof.

Lemma 7.3. Let $w \in L_{\text {loc }}^{1}\left(\mathbb{R}^{N}\right) \cap B V\left(\mathbb{R}^{N}\right)$ and $\eta: \mathbb{R} \rightarrow \mathbb{R}$ Lipschitz-continuous, with a Lipschitz constant equal to 1 . Then, $\eta(w) \in B V\left(\mathbb{R}^{N}\right)$ and its total variation satisfies $|D(\eta(w))| \leq|D w|$.

Proof of Lemma 7.3. Consider $\varphi_{\mu}=\rho_{\mu} * w$, where $\rho_{\mu}$ is an approximate unit as before. The smooth functions $\varphi_{\mu}$ converge to $w$ as $\mu \rightarrow 0$ and in $L_{l o c}^{1}\left(\mathbb{R}^{N}\right)$, with gradients bounded in $L^{1}$ norm by $|w|_{B V\left(\mathbb{R}^{N}\right)}$. Since $\eta\left(\varphi_{\mu}\right) \rightarrow \eta(w)$ in $L_{\text {loc }}^{1}\left(\mathbb{R}^{N}\right)$ and a fortiori in $\mathcal{D}^{\prime}\left(\mathbb{R}^{N}\right)$, the gradients converge in $\mathcal{D}^{\prime}\left(\mathbb{R}^{N}, \mathbb{R}^{N}\right)$. Moreover, $\nabla\left(\eta\left(\varphi_{\mu}\right)\right)=\eta^{\prime}\left(\varphi_{\mu}\right) \nabla \varphi_{\mu}$. Then, $\left\|\nabla\left(\eta\left(\varphi_{\mu}\right)\right)\right\|_{\left(L^{1}\left(\mathbb{R}^{N}\right)\right)^{N}} \leq$ $|w|_{B V\left(\mathbb{R}^{N}\right)}$ and Banach-Alaoglu-Bourbaki's Theorem implies that $\nabla\left(\eta\left(\varphi_{\mu}\right)\right)$ converges to any $\vartheta \in M_{b}\left(\mathbb{R}^{N}, \mathbb{R}^{N}\right)$, up to a subsequence, for the weak convergence of measures. Identifying the limit in $\mathcal{D}^{\prime}\left(\mathbb{R}^{N}, \mathbb{R}^{N}\right), \vartheta=D(\eta(w))$ and we already know that $\eta(w) \in B V\left(\mathbb{R}^{N}\right)$. Moreover, for all $F \in C_{0}\left(\mathbb{R}^{N}, \mathbb{R}^{N}\right)$

$$
\int_{\mathbb{R}^{N}} F \cdot \nabla\left(\eta\left(\varphi_{\mu}\right)\right) \leq \int_{\mathbb{R}^{N}}|F|\left|\nabla \varphi_{\mu}\right|
$$

Since $\nabla \varphi_{\mu}=\rho_{\mu} * D w,\left|\nabla \varphi_{\mu}\right| \leq \rho_{\mu} *|D w|$ and the limit $\mu \rightarrow 0$ implies that for all $F \in C_{0}\left(\mathbb{R}^{N}, \mathbb{R}^{N}\right)$, $\int_{\mathbb{R}^{N}} F d D(\eta(w)) \leq \int_{\mathbb{R}^{N}}|F| d|D w|$. The proof is now complete.

\subsection{Proof of Proposition 2.1 item iii)}

We begin by a technical lemma that will be needed.

Lemma 7.4. Let $u \in L^{\infty}(] 0, \infty\left[\times \mathbb{R}^{N}\right)$ and $\theta_{\nu}$ be an approximate unit as before. Let us assume that $\theta_{\nu}$ is of the form $\theta_{\nu}=\nu^{-1} \theta\left(\nu^{-1}\right.$.). Then, for a.e. $\left.t \in\right] 0, \infty[$

i) $u(t,$.$) is a measurable function essentially bounded by \|u\|_{L^{\infty}(] 0, \infty\left[\times \mathbb{R}^{N}\right)}$;

ii) for all $\gamma \in C_{c}^{\infty}\left(\mathbb{R}^{N}\right), \lim _{\nu \rightarrow 0} \int_{0}^{\infty} \int_{\mathbb{R}^{N}} u(s, x) \theta_{\nu}(s-t) \gamma(x) d x d s=\int_{\mathbb{R}^{N}} u(t,.) \gamma$. 
Proof. Let $D$ be a countable dense subset of $C_{c}^{\infty}\left(\mathbb{R}^{N}\right)$ for the topology of the $L^{1}$-norm. The lemma above, with ii) satisfied for all $\gamma \in D$, is an immediate consequence of Fubini's Theorem (in particular, for the measurability mentioned in i)) and of the existence of the Lebesgue's points of the locally integrable functions $s \rightarrow \int_{\mathbb{R}^{N}} u(s,.) \gamma$ (for all $\gamma \in D$ ). For $\gamma \in C_{c}^{\infty}\left(\mathbb{R}^{N}\right)$ and any sequence $\left(\gamma_{n}\right)_{n} \subseteq D$ which converges to $\gamma$ in $L^{1}\left(\mathbb{R}^{N}\right)$, we have

$$
\int_{0}^{\infty} \int_{\mathbb{R}^{N}} u(s, x) \theta_{\nu}(s-t) \gamma_{n}(x) d x d s \rightarrow \int_{0}^{\infty} \int_{\mathbb{R}^{N}} u(s, x) \theta_{\nu}(s-t) \gamma(x) d x d s
$$

as $n \rightarrow+\infty$ and independently on $\nu>0$. The theorem of inversion of the limits then implies ii) for all $\gamma \in C_{c}^{\infty}\left(\mathbb{R}^{N}\right)$ and this completes the proof of Lemma 7.4.

Let us return to the proof of Proposition 2.1 item iii). In the sequel, we let dom $u$ denote the set of $t \in] 0, \infty[$ such that the items i) and ii) of the lemma above hold true.

First step. Let us prove that $u$ is Lipschitz-continuous on $\left[0, \infty\left[\right.\right.$ with values in $L^{\infty}\left(\mathbb{R}^{N}\right)$, endowed with the weak- $\star$ topology. Let $t_{1}$ and $t_{2}$ belong to dom $u$. Let us assume without loss of generality that $t_{2}>t_{1}$. Consider test-functions of the form

$$
\varphi(t, x)=\left(\int_{0}^{t} \theta_{\nu}\left(s-t_{1}\right)-\theta_{\nu}\left(s-t_{2}\right) d s\right) \gamma(x) .
$$

Let us take $\varphi$ in the weak formulation of Proposition 2.1 item ii) and, thanks to Lemma 7.4 ii), let us pass to the limit as $\nu \rightarrow 0$. We get

$$
\int_{\mathbb{R}^{N}}\left(u\left(t_{2}, .\right)-u\left(t_{1}, .\right)\right) \gamma=\int_{t_{1}}^{t_{2}} \int_{\mathbb{R}^{N}}(f(u) . \nabla \gamma-u g[\gamma]) \leq C\|\gamma\|_{W^{2,1}\left(\mathbb{R}^{N}\right)}\left(t_{2}-t_{1}\right),
$$

where $C$ is any constant that only depends on the $L^{\infty}$-norm of $u$ and the flux $f$ (in the sequel, we still let $C$ denote such a constant). Taking the supremum w.r.t. $\gamma \in C_{c}^{\infty}\left(\mathbb{R}^{N}\right)$ such that $\|\gamma\|_{W^{2,1}\left(\mathbb{R}^{N}\right)} \leq 1$, we deduce that

$$
\left\|u\left(t_{2}, .\right)-u\left(t_{1}, .\right)\right\|_{W^{2,1^{\prime}}\left(\mathbb{R}^{N}\right)} \leq C\left|t_{2}-t_{1}\right|,
$$

for all $t_{1}, t_{2} \in \operatorname{dom} u$. Then, $u$ can be continuously extended in a unique way as a continuous function from $\left[0, \infty\left[\right.\right.$ into $W^{2,1^{\prime}}\left(\mathbb{R}^{N}\right)$, since it is a Lipschitz continuous function on a dense subset (indeed, the Lebesgue measure of $[0, \infty[\backslash \operatorname{dom} u$ is null). Banach-Aloaglu-Bourbaki's Theorem then implies that this extension is with values in $L^{\infty}\left(\mathbb{R}^{N}\right)$ and is continuous on $[0, \infty[$ for the weak- $\star$ topology.

Second step. Let us prove that $u:\{0\} \cup\left(\operatorname{dom} u \cap \operatorname{dom} u^{2}\right) \rightarrow L_{l o c}^{1}\left(\mathbb{R}^{N}\right)$ is continuous from the right. Let us now assume that $t_{i}$ belongs to $\operatorname{dom} u \cap \operatorname{dom} u^{2}(i=1,2)$ with $t_{2}>t_{1}$. Consider the entropy $\eta=|.|^{2}$ and $\phi$ any flux associated to $\eta$. As noted in Remark 4.2, the entropy solution $u$ of (1.1) is a fortiori an intermediate solution and arguing as in the preceding step by using (4.17), we get

$$
\int_{\mathbb{R}^{N}} u^{2}\left(t_{2}, .\right) \gamma \leq \int_{\mathbb{R}^{N}} u^{2}\left(t_{1}, .\right) \gamma+C\|\gamma\|_{W^{2,1}\left(\mathbb{R}^{N}\right)}\left(t_{2}-t_{1}\right)
$$

for all nonnegative $\gamma \in C_{c}^{\infty}\left(\mathbb{R}^{N}\right)$. Simple computations then show that

$$
\int_{\mathbb{R}^{N}}\left(u\left(t_{2}, .\right)-u\left(t_{1}, .\right)\right)^{2} \gamma=\int_{\mathbb{R}^{N}} u^{2}\left(t_{2}, .\right) \gamma+\int_{\mathbb{R}^{N}} u^{2}\left(t_{1}, .\right) \gamma-2 \int_{\mathbb{R}^{N}} u\left(t_{2}, .\right) u\left(t_{1}, .\right) \gamma,
$$


for all nonnegative $\gamma \in C_{c}^{\infty}\left(\mathbb{R}^{N}\right)$. Since $u\left(t_{1},.\right) \gamma \in L^{1}\left(\mathbb{R}^{N}\right)$, the preceding step and (7.5) implies that $u\left(t_{2},.\right)$ converges to $u\left(t_{1},.\right)$ in $L_{l o c}^{2}\left(\mathbb{R}^{N}\right)$ as dom $u \cap \operatorname{dom} u^{2} \ni t_{2} \rightarrow t_{1}^{+}$. Moreover, similar arguments with test-functions of the form $\varphi(t, x)=\int_{t}^{\infty} \theta_{\nu}(s-T) d s \gamma(x)\left(T \in \operatorname{dom} u \cap \operatorname{dom} u^{2}\right)$ allow to prove that $u(T,$.$) converges to u_{0}$ in $L_{\text {loc }}^{1}\left(\mathbb{R}^{N}\right)$ as $\operatorname{dom} u \cap \operatorname{dom} u^{2} \ni T \rightarrow 0$.

Conclusion. Let us prove that $\left\{u(t,):. t \in \operatorname{dom} u \cap \operatorname{dom} u^{2}\right\}$ is relatively compact in $L_{l o c}^{1}\left(\mathbb{R}^{N}\right)$, thus concluding that $u \in C\left(\left[0, \infty\left[; L_{l o c}^{1}\left(\mathbb{R}^{N}\right)\right)\right.\right.$ with the help of the first step. We can argue exactly as in Section 4 to establish (3.1) for all entropy solutions $u$ and $v$ of (1.1) and all $t \in \operatorname{dom} u \cap \operatorname{dom} v$. Indeed, the continuity property of the second step suffices to prove (4.10) and (4.15), only points where the continuity with values in $L_{l o c}^{1}\left(\mathbb{R}^{N}\right)$ of entropy solutions has been used. Then, we can get an estimate on the translations of $u(t,$.$) and by the essentially bound mentioned in the item$ i) of Lemma 7.4, Kolmogorov's Theorem implies that $\left\{u(t,):. t \in \operatorname{dom} u \cap \operatorname{dom} u^{2}\right\}$ is relatively compact in $L_{l o c}^{1}\left(\mathbb{R}^{N}\right)$. Thus, the proof of Proposition 2.1 iii) is complete.

\section{Acknowledgments}

The author would like to thank J. Vovelle for the fruitful discussions we had together.

\section{References}

[1] P. Biler, T. Funaki and W. A. Woyczyński, Fractal Burgers Equations, Journal of Differenial Equations 148 (1998), no. 1, 9-46.

[2] P. Biler, G. Karch and W. A. Woyczyński, Critical nonlinearity exponent and self-similar asymptotics for Lev25 conservation laws, Ann. Inst. H. Poincaré Anal. NonLinéaire 18 (2001), no. 5, 613-637.

[3] P. Clavin and B. Denet, Theory of cellular detonations in gases, part 2. Mach-stem formation at strong overdrive, C. R. Acad. Sci. Paris t. 329 (2001), no. Série II b, 489-496.

[4] P. Clavin and L. He, Theory of cellular detonations in gases, part 1., Stability limits at strong overdrive, C. R. Acad. Sci. Paris t. 329 (2001), no. Série II b, 463-471.

[5] J. Carrillo, Entropy Solutions for Nonlinear Degenerate Problems, Archive for Rational Mechanics \& Analysis, 147 (1999), 269-361.

[6] J. Droniou, Vanishing non-local regularization of a scalar conservation law, Electronic Journal of Differential Equations, 2003 (2003), no. 117, 1-20.

[7] J. Droniou, T. Gallouët and J. Vovelle, Global solution and smoothing effect for a non-local regularization of an hyperbolic equation, Journal of Evolution Equations, 3 (2003), no. 3, 499-521, Dedicated to Philippe Bénilan.

[8] J. Droniou and C. Imbert, Fractal first order partial differential equations, to appear in Archive for Rational Mechanics \& Analysis.

[9] C. Imbert, A non-local regularization of first order Hamilton-Jacobi equations, Journal of Differential Equations 211 (2005), 214-246.

[10] B. Jourdain, S. Méléard and W. A. Woyczyński, Probalistic approximation and inviscid limits for 1-D fractional conservation laws, Bernoulli, 11 (2005), no. 4, 689-714. 
[11] S. N. Kruzhkov, First order quasilinear equations with several independent variables, Math. Sb. (N.S.) 81 (123) (1970), 228-255.

[12] N. N. Kuznecov, The accuracy of certain approximate methods for the computation of weak solutions of a first order quasilinear equations, Ž. Vyčisl. Mat. i Mat. Fiz, 16 (1976), no. 1627, 1489-1502.

[13] O. A. Ladyzenskaja, V. A. Solonnikov and N. N. Ural'čeva, Linear and Quasilinear Equations of Parabolic Type, Translations of Math. Monographs, 23 (1967), 1489-1502.

[14] P. Lévy, Calcul des Probabilités, (1925). 